\title{
Bi-unitary multiperfect numbers, IV(a)
}

\author{
Pentti Haukkanen ${ }^{1}$ and Varanasi Sitaramaiah $^{2, *}$ \\ ${ }^{1}$ Faculty of Information Technology and Communication Sciences, \\ FI-33014 Tampere University, Finland \\ e-mail: pentti.haukkanen@tuni.fi \\ 2 1/194e, Poola Subbaiah Street, Taluk Office Road, Markapur \\ Prakasam District, Andhra Pradesh, 523316 India
}

Dedicated to the memory of Prof. D. Suryanarayana

\begin{abstract}
A divisor $d$ of a positive integer $n$ is called a unitary divisor if $\operatorname{gcd}(d, n / d)=1$; and $d$ is called a bi-unitary divisor of $n$ if the greatest common unitary divisor of $d$ and $n / d$ is unity. The concept of a bi-unitary divisor is due to D. Surynarayana (1972). Let $\sigma^{* *}(n)$ denote the sum of the bi-unitary divisors of $n$. A positive integer $n$ is called a bi-unitary multiperfect number if $\sigma^{* *}(n)=k n$ for some $k \geq 3$. For $k=3$ we obtain the bi-unitary triperfect numbers.

Peter Hagis (1987) proved that there are no odd bi-unitary multiperfect numbers. The present paper is Part IV(a) in a series of papers on even bi-unitary multiperfect numbers. In parts I, II and III we found all bi-unitary triperfect numbers of the form $n=2^{a} u$, where $1 \leq a \leq 6$ and $u$ is odd. There exist exactly ten such numbers. In this part we solve partly the case $a=7$. We prove that if $n$ is a bi-unitary triperfect number of the form $n=2^{7} \cdot 5^{b} \cdot 17^{c} . v$, where $(v, 2.5 .17)=1$, then $b \geq 2$. We then confine ourselves to the case $b=2$. We prove that in this case we have $c=1$ and further show that $n=2^{7} \cdot 3^{2} \cdot 5^{2} \cdot 7 \cdot 13 \cdot 17=44553600$ is the only bi-unitary triperfect number of this form.
\end{abstract}

Keywords: Perfect numbers, Triperfect numbers, Multiperfect numbers, Bi-unitary analogues. 2010 Mathematics Subject Classification: 11A25.

*Prof. Varanasi Sitaramaiah passed away on 2 October 2020. 


\section{Introduction}

Throughout this paper, all lower case letters denote positive integers; $p$ and $q$ denote primes. The letters $u, v$ and $w$ are reserved for odd numbers.

A divisor $d$ of $n$ is called a unitary divisor if $\operatorname{gcd}(d, n / d)=1$. If $d$ is a unitary divisor of $n$, we write $d \| n$. A divisor $d$ of $n$ is called a bi-unitary divisor if $(d, n / d)^{* *}=1$, where the symbol $(a, b)^{* *}$ denotes the greatest common unitary divisor of $a$ and $b$. The concept of a bi-unitary divisor is due to D. Suryanarayana (cf. [6]). Let $\sigma^{* *}(n)$ denote the sum of bi-unitary divisors of $n$. The function $\sigma^{* *}(n)$ is multiplicative, that is, $\sigma^{* *}(1)=1$ and $\sigma^{* *}(m n)=\sigma^{* *}(m) \sigma^{* *}(n)$ whenever $(m, n)=1$. If $p^{\alpha}$ is a prime power and $\alpha$ is odd, then every divisor of $p^{\alpha}$ is a bi-unitary divisor; if $\alpha$ is even, each divisor of $p^{\alpha}$ is a bi-unitary divisor except for $p^{\alpha / 2}$. Hence

$$
\sigma^{* *}\left(p^{\alpha}\right)=\left\{\begin{array}{l}
\sigma\left(p^{\alpha}\right)=\frac{p^{\alpha+1}-1}{p-1} \text { if } \alpha \text { is odd }, \\
\sigma\left(p^{\alpha}\right)-p^{\alpha / 2} \text { if } \alpha \text { is even. }
\end{array}\right.
$$

If $\alpha$ is even, say $\alpha=2 k$, then $\sigma^{* *}\left(p^{\alpha}\right)$ can be simplified to

$$
\sigma^{* *}\left(p^{\alpha}\right)=\left(\frac{p^{k}-1}{p-1}\right) \cdot\left(p^{k+1}+1\right) .
$$

From (1.3), it is not difficult to observe that $\sigma^{* *}(n)$ is odd only when $n=1$ or $n=2^{\alpha}$.

The concept of a bi-unitary perfect number was introduced by C. R. Wall [7]; a positive integer $n$ is called a bi-unitary perfect number if $\sigma^{* *}(n)=2 n$. C. R. Wall [7] proved that there are only three bi-unitary perfect numbers, namely 6,60 and 90 . A positive integer $n$ is called a bi-unitary multiperfect number if $\sigma^{* *}(n)=k n$ for some $k \geq 3$. For $k=3$ we obtain the bi-unitary triperfect numbers.

Peter Hagis [1] proved that there are no odd bi-unitary multiperfect numbers. Our present paper is Part IV(a) in a series of papers on even bi-unitary multiperfect numbers. In Part I (see [2]), we found all bi-unitary triperfect numbers of the form $n=2^{a} u$, where $1 \leq a \leq 3$ and $u$ is odd. We proved that if $1 \leq a \leq 3$ and $n=2^{a} u$ is a bi-unitary triperfect number, then $a=3$ and $n=120=2^{3}$.3.5. In Part II (see [3]), we considered the cases $a=4$ and $a=5$. We proved that if $n=2^{4} u$ is a bi-unitary triperfect number, then $n=2160=2^{4} .3^{3} .5$, and that if $n=2^{5} u$ is a bi-unitary triperfect number, then $n=672=2^{5} .3 .7, n=10080=2^{5} .3^{2} .5 .7$, $n=528800=2^{5} .3 .5^{2} .13$ or $n=22932000=2^{5} \cdot 3^{2} \cdot 5^{3} .7^{2} .13$. In Part III (see [4]) we showed that the bi-unitary triperfect numbers of the form $n=2^{6} u$ are $n=22848=2^{6} .3 .7 .17, n=342720=$ $2^{6} .3^{2} .5 .7 .17, n=51979200=2^{6} \cdot 3 \cdot 5^{2} \cdot 7^{2} \cdot 13.17$ and $n=779688000=2^{6} .3^{2} \cdot 5^{3} \cdot 7^{2} .13 .17$. In the present part we consider the case $a=7$; we solve it partly. We prove that if $n$ is a bi-unitary triperfect number of the form $n=2^{7} \cdot 5^{b} .17^{c} . v$, where $(v, 2.5 .17)=1$, then $b \geq 2$. We then confine ourselves to the case $b=2$. We prove that in this case $c$ has to equal 1 and further show that $n=2^{7} \cdot 3^{2} \cdot 5^{2} \cdot 7 \cdot 13.17=44553600$ is the only bi-unitary triperfect number of the form considered here. We will continue the study of the case $a=7$ in future papers.

For a general account on various perfect-type numbers, we refer to [5]. 


\section{Preliminaries}

We assume that the reader has Part I (see [2]) available. We, however, recall Lemmas 2.1 to 2.4 from Part I, because they are so important also here.

Lemma 2.1. (I) If $\alpha$ is odd, then

$$
\frac{\sigma^{* *}\left(p^{\alpha}\right)}{p^{\alpha}}>\frac{\sigma^{* *}\left(p^{\alpha+1}\right)}{p^{\alpha+1}}
$$

for any prime $p$.

(II) For any $\alpha \geq 2 \ell-1$ and any prime $p$,

$$
\frac{\sigma^{* *}\left(p^{\alpha}\right)}{p^{\alpha}} \geq\left(\frac{1}{p-1}\right)\left(p-\frac{1}{p^{2 \ell}}\right)-\frac{1}{p^{\ell}}=\frac{1}{p^{2 \ell}}\left(\frac{p^{2 \ell+1}-1}{p-1}-p^{\ell}\right) .
$$

(III) If $p$ is any prime and $\alpha$ is a positive integer, then

$$
\frac{\sigma^{* *}\left(p^{\alpha}\right)}{p^{\alpha}}<\frac{p}{p-1}
$$

Remark 2.1. (I) and (III) of Lemma 2.1 are mentioned in C. R. Wall [7]; (II) of Lemma 2.1 has been used by him [7] without explicitly stating it.

Lemma 2.2. Let $a>1$ be an integer not divisible by an odd prime $p$ and let $\alpha$ be a positive integer. Let $r$ denote the least positive integer such that $a^{r} \equiv 1\left(\bmod p^{\alpha}\right) ;$ then $r$ is usually denoted by $\operatorname{ord}_{p^{\alpha}}$ a. We have the following properties.

(i) If $r$ is even, then $s=r / 2$ is the least positive integer such that $a^{s} \equiv-1\left(\bmod p^{\alpha}\right)$. Also, $a^{t} \equiv-1\left(\bmod p^{\alpha}\right)$ for a positive integer $t$ if and only if $t=s u$, where $u$ is odd.

(ii) If $r$ is odd, then $p^{\alpha} \nmid a^{t}+1$ for any positive integer $t$.

Remark 2.2. Let $a, p, r$ and $s=r / 2$ be as in Lemma $2.2(\alpha=1)$. Then $p \mid a^{t}-1$ if and only if $r \mid t$. If $t$ is odd and $r$ is even, then $r \nmid t$. Hence $p \nmid a^{t}-1$. Also, $p \mid a^{t}+1$ if and only if $t=s u$, where $u$ is odd. In particular if $t$ is even and $s$ is odd, then $p \nmid a^{t}+1$. In order to check the divisibility of $a^{t}-1$ (when $t$ is odd) by an odd prime $p$, we can confine to those $p$ for which $\operatorname{ord}_{p} a$ is odd. Similarly, for examining the divisibility of $a^{t}+1$ by $p$ when $t$ is even we need to consider primes $p$ with $s=\operatorname{ord}_{p} a / 2$ even.

Lemma 2.3. Let $k$ be odd and $k \geq 3$. Let $p \neq 5$.

(a) If $p \in[3,2520]-\{11,19,31,71,181,829,1741\}$, ord $d_{p} 5$ is odd and $p \mid 5^{k}-1$, then we can find a prime $p^{\prime}$ (depending on $p$ ) such that $p^{\prime} \mid \frac{5^{k}-1}{4}$ and $p^{\prime} \geq 2521$.

(b) If $q \in[3,2520]-\{13,313,601\}, s=\frac{1}{2} \operatorname{ord}_{q} 5$ is even and $q \mid 5^{k+1}+1$, then we can find a prime $q^{\prime}$ (depending on $q$ ) such that $q^{\prime} \mid \frac{5^{k+1}+1}{2}$ and $q^{\prime} \geq 2521$.

Lemma 2.4. Let $k$ be odd and $k \geq 3$. Let $p \neq 7$.

(a) If $p \in[3,2520]-\{3,19,37,1063\}, r=\operatorname{ord}_{p} 7$ is odd and $p \mid 7^{k}-1$, then we can find a prime $p^{\prime}$ (depending on $p$ ) such that $p^{\prime} \mid \frac{7^{k}-1}{6}$ and $p^{\prime} \geq 2521$.

(b) If $q \in[3,1193]-\{5,13,181,193,409\}, s=\frac{1}{2} \operatorname{ord}_{q} 7$ is even and $q \mid 7^{k+1}+1$, then we can find a prime $q^{\prime}$ (depending on $q$ ) such that $q^{\prime} \mid \frac{7^{k+1}+1}{2}$ and $q^{\prime}>1193$. 
Lemma 2.5. Let $k$ be odd and $k \geq 3$. Let $p \neq 13$.

(a) If $p \in[3,293]-\{3,61\}, r=\operatorname{ord}_{p} 13$ is odd and $p \mid 13^{k}-1$, then we can find a prime $p^{\prime}$ (depending on $p$ ) such that $p^{\prime} \mid \frac{13^{k}-1}{12}$ and $p^{\prime} \geq 293$.

(b) If $q \in[3,293]-\{5,17\}, s=\frac{1}{2} \operatorname{ord}_{q} 13$ is even and $q \mid 13^{k+1}+1$, then we can find a prime $q^{\prime}$ (depending on $q$ ) such that $q^{\prime} \mid \frac{13^{k+1}+1}{2}$ and $q^{\prime}>293$.

Proof. (a) Let $p \mid 13^{k}-1$. If $r=\operatorname{ord}_{p} 13$, that is, $r$ is the least positive integer such that $13^{r} \equiv 1(\bmod p)$, then $r \mid k$. Since $k$ is odd, $r$ must be odd. Also, $13^{r}-1 \mid 13^{k}-1$. Let

$$
S_{13}=\left\{(p, r): p \neq 13, p \in[3,293] \text { and } r=\operatorname{ord}_{p} 13 \text { is odd }\right\} .
$$

From Appendix A, we have

$$
\begin{aligned}
S_{13}= & (3,1),(23,11),(43,21),(53,13),(61,3),(79,39),(103,17), \\
& (107,53),(127,63),(131,65),(139,69),(179,89),(181,45), \\
& (191,95),(199,99),(211,35),(251,125),(263,131),(283,141)\} .
\end{aligned}
$$

Let $p \mid 13^{k}-1$ and $p \in[3,293]-\{3,61\}$. Then $(p, r) \in S_{13}-\{(3,1),(61,3)\}$, where $r=$ ord $d_{p} 13$. Also, $13^{r}-1 \mid 13^{k}-1$. To prove (a), it is enough to show that $\frac{13^{r}-1}{12}$ is divisible by a prime $p^{\prime} \geq 293$. From Appendix C, we know the factors of $13^{r}-1$. By examining the factors of $13^{r}-1$ for $r \notin\{1,3\}$, which correspond to the primes 3 and 61 , we infer that we can find a prime $p^{\prime}\left|\frac{13^{r}-1}{12}\right| \frac{13^{k}-1}{12}$ satisfying $p^{\prime} \geq 293$. This proves (a).

For example, if $p=43$, then $r=21$. Also,

$$
13^{21}-1=\{\{2,2\},\{3,2\},\{43,1\},\{61,1\},\{337,1\},\{547,1\},\{2714377,1\},\{5229043,1\}\} .
$$

We can take $p^{\prime}=337$.

(b) Let $q \mid 13^{k+1}+1$ and $q \in[3,293]-\{5,17\}$. Let $r=\operatorname{ord}_{q} 13$. If $r$ is odd, then $q \nmid 13^{k+1}+1$ (see Remark $2.2(a=13))$. We may assume that $r$ is even. Let $s=r / 2$. Then $s$ is the least positive integer such that $q \mid 13^{s}+1$. Again from Remark $2.2(a=13), q \nmid 13^{k+1}+1$ if $s$ is odd. Since $q \mid 13^{k+1}+1$, we have that $s$ is even. Also, $k+1=s u$, where $u$ is odd. This implies that $13^{s}+1 \mid 13^{k+1}+1$. Let

$$
T_{13}=\left\{(q, s): q \neq 13, q \in[3,293] \text { and } s=\frac{1}{2} \operatorname{ord}_{q} 13 \text { even }\right\} .
$$

From Appendix A, we have

$$
\begin{aligned}
T_{13}= & (5,2),(17,2),(37,18),(41,20),(73,36),(89,44), \\
& (97,48),(109,54),(113,28),(137,68),(149,74),(193,32), \\
& (197,98),(229,38),(233,58),(241,120),(257,64),(281,140),(293,146)\} .
\end{aligned}
$$

Let $q \mid 13^{k+1}+1$ and $q \in[3,293]-\{5,17\}$. Then $(q, s) \in T_{13}-\{(5,2),(17,2)\}$, where $s=\frac{1}{2} \operatorname{ord}_{q} 13$. To prove (b), it is enough to show that $\frac{13^{s}+1}{2}$ is divisible by a prime $q^{\prime}>293$ 
for all $s \in T_{13}^{\prime}=\left\{s:(q, s) \in T_{13}-\{(5,2),(17,2)\}\right.$. This follows by examining the factors of $13^{s}+1$ given in Appendix D.

For example, if $q=37$, then $s=18$. Also,

$$
13^{18}+1=\{\{2,1\},\{5,1\},\{17,1\},\{37,1\},\{28393,1\},\{428041,1\},\{1471069,1\}\} .
$$

We can take $q^{\prime}=28393$.

Lemma 2.6. Let $k$ be odd and $k \geq 3$. Let $p \neq 17$.

(a) If $p \in[3,519]-\{307\}, r=\operatorname{ord}_{p} 17$ is odd and $p \mid 17^{k}-1$, then we can find a prime $p^{\prime}$ (depending on $p$ ) such that $p^{\prime} \mid \frac{17^{k}-1}{16}$ and $p^{\prime}>519$.

(b) If $q \in[3,519]-\{5,29\}, s=\frac{1}{2} \operatorname{ord}_{q} 17$ is even and $q \mid 17^{k+1}+1$, then we can find a prime $q^{\prime}$ (depending on $q$ ) such that $q^{\prime} \mid \frac{17^{k+1}+1}{2}$ and $q^{\prime}>519$.

Proof. (a) Let $p \mid 17^{k}-1$. If $r=\operatorname{ord}_{p} 17$, that is, $r$ is the least positive integer such that $17^{r} \equiv 1(\bmod p)$, then $r \mid k$. Since $k$ is odd, $r$ must be odd. Also, $17^{r}-1 \mid 17^{k}-1$. Let

$$
S_{17}=\left\{(p, r): p \neq 17, p \in[3,519] \text { and } r=\operatorname{ord}_{p} 17 \text { is odd }\right\} .
$$

From Appendix B, we have

$$
\begin{aligned}
S_{17}= & \{(19,9),(43,21),(47,23),(59,29),(67,33),(83,41),(103,51), \\
& (127,63),(149,37),(151,75),(157,39),(179,89),(191,95),(223,37), \\
& (229,19),(239,119),(263,131),(271,135),(293,73),(307,3),(331,165), \\
& (359,179),(383,191),(389,97),(409,51),(433,27),(443,221), \\
& (463,231),(467,233),(491,49),(509,127)\} .
\end{aligned}
$$

Let $p \mid 17^{k}-1$ and $p \in[3,519]-\{307\}$. Then $(p, r) \in S_{17}-\{(307,3)\}$, where $r=\operatorname{ord}_{p} 17$. Also, $17^{r}-1 \mid 17^{k}-1$. To prove (a), it is enough to show that $\frac{17^{r}-1}{16}$ is divisible by a prime $p^{\prime} \geq 519$. From Appendix E, we know the factors of $17^{r}-1$. By examining the factors of $17^{r}-1$ for $r \notin\{3\}$, which corresponds to the prime 307 , we infer that we can find a prime $p^{\prime}\left|\frac{17^{r}-1}{16}\right| \frac{17^{k}-1}{16}$ satisfying $p^{\prime}>519$. This proves (a).

For example, if $p=19$, then $r=9$. Also,

$$
17^{9}-1=\{\{2,4\},\{19,1\},\{307,1\},\{1270657,1\}\} .
$$

We can take $p^{\prime}=1270657$.

(b) Let $q \mid 17^{k+1}+1$ and $q \in[3,519]-\{5,29\}$. Let $r=\operatorname{ord}_{q} 17$. If $r$ is odd, then $q \nmid 17^{k+1}+1$ (see Remark $2.2(a=17)$ ). We may assume that $r$ is even. Let $s=r / 2$. Then $s$ is the least positive integer such that $q \mid 17^{s}+1$. Again from Remark $2.2(a=17), q \nmid 17^{k+1}+1$ if $s$ is odd. Since $q \mid 17^{k+1}+1$, we have that $s$ is even. Also, $k+1=s u$, where $u$ is odd. This implies that $17^{s}+1 \mid 17^{k+1}+1$. Let

$$
T_{17}=\left\{(q, s): q \neq 17, q \in[3,519] \text { and } s=\frac{1}{2} \operatorname{ord}_{q} 17 \text { even }\right\} .
$$


From Appendix B, we have

$$
\begin{aligned}
T_{17}= & \{(5,2),(29,2),(37,18),(41,20),(61,30),(73,12),(89,22),(97,48),(109,18), \\
& (113,56),(137,34),(173,86),(181,18),(193,96),(197,98),(233,116),(241,40), \\
& (257,16),(269,134),(277,138),(281,70),(313,156),(317,158),(337,56), \\
& (353,44),(397,66),(401,200),(449,224)\} .
\end{aligned}
$$

Let $q \mid 17^{k+1}+1$ and $q \in[3,519]-\{5,29\}$. Then $(q, s) \in T_{17}-\{(5,2),(29,2)\}$, where $s=\frac{1}{2} \operatorname{ord}_{q} 17$. To prove (b), it is enough to show that $\frac{17^{s}+1}{2}$ is divisible by a prime $q^{\prime}>519$ for all $s \in T_{17}^{\prime}=\left\{s:(q, s) \in T_{17}-\{(5,2),(29,2)\}\right.$. This follows by examining the factors of $17^{s}+1$ given in Appendix F.

For example if $q=37$, then $s=18$. Also,

$$
\begin{aligned}
17^{18}+1= & \{\{2,1\},\{5,1\},\{29,1\},\{37,1\},\{109,1\},\{181,1\}, \\
& \{2089,1\},\{83233,1\},\{382069,1\}\} .
\end{aligned}
$$

We can take $q^{\prime}=2089$.

\section{Partial results on bi-unitary triperfect numbers of the form $n=2^{7} u$}

Let $n$ be a bi-unitary triperfect number divisible unitarily by $2^{7}$ so that $\sigma^{* *}(n)=3 n$ and $n=2^{7}$. $u$, where $u$ is odd. Since $\sigma^{* *}\left(2^{7}\right)=2^{8}-1=255=3.5 .17$, using $n=2^{7} u$ in $\sigma^{* *}(n)=3 n$, we get the following equations:

$$
n=2^{7} \cdot 5^{b} \cdot 17^{c} \cdot v
$$

and

$$
2^{7} \cdot 5^{b-1} \cdot 17^{c-1} \cdot v=\sigma^{* *}\left(5^{b}\right) \cdot \sigma^{* *}\left(17^{c}\right) \cdot \sigma^{* *}(v),
$$

where $(v, 2.5 .17)=1$. Considering the parity of the function values of $\sigma^{* *}$ and applying multiplicativity of $\sigma^{* *}$ we conclude that $v$ has not more than five odd prime factors. Also note that $b, c \geq 1$.

In this paper we show that $b \geq 2$ in $(3.1 a)$ and consider completely the case $b=2$. We will examine the case $b \geq 3$ in future papers.

Theorem 3.1. (a) If $n$ is as in (3.1a) and $n$ is a bi-unitary triperfect number, then $b \geq 2$. (b) If $b=2$, then $c=1$ and $n=44553600=2^{7} \cdot 3^{2} \cdot 5^{2} \cdot 7 \cdot 13.17$.

Proof. (a) We assume that $b=1$ and obtain a contradiction. Since $\sigma^{* *}(5)=6$, taking $b=1$ in $(3.1 b)$, after simplification we get

$$
2^{6} \cdot 17^{c-1} \cdot v=3 \cdot \sigma^{* *}\left(17^{c}\right) \cdot \sigma^{* *}(v) .
$$

From (3.2), 3|v. Let $v=3^{d} . w$, where $(w, 2.3 .5 .17)=1$. 
From $(3.1 a)$ we have

$$
n=2^{7} \cdot 5 \cdot 17^{c} \cdot 3^{d} \cdot w
$$

and from (3.2),

$$
2^{6} \cdot 17^{c-1} \cdot 3^{d-1} \cdot w=\sigma^{* *}\left(17^{c}\right) \cdot \sigma^{* *}\left(3^{d}\right) \cdot \sigma^{* *}(w),
$$

$w$ has not more than four odd prime factors and $(w, 2.3 .5 .17)=1$.

If $d=1$, from $(3.2 a)$, we have, by (1.3),

$$
3=\frac{\sigma^{* *}(n)}{n} \geq \frac{255}{128} \cdot \frac{6}{5} \cdot \frac{4}{3}=3.1875>3
$$

a contradiction.

Taking $d=2$ in $(3.2 b)$, since $\sigma^{* *}\left(3^{2}\right)=10$, we see that $5 \mid w$. But this is false. Hence $d \neq 2$.

Thus we may assume that $d \geq 3$. By Lemma 2.1, $\frac{\sigma^{* *}\left(3^{d}\right)}{3^{d}} \geq \frac{112}{81}$. Hence from $(3.2 a)$,

$$
3=\frac{\sigma^{* *}(n)}{n} \geq \frac{255}{128} \cdot \frac{6}{5} \cdot \frac{112}{81}=3.3>3,
$$

a contradiction.

Hence $b=1$ is not admissible. Hence $b \geq 2$.

The proof of (a) is complete.

(b) Since $\sigma^{* *}\left(5^{2}\right)=26=2.13$, taking $b=2$ in $(3.1 b)$, we find that $13 \mid v$. Let $v=13^{d}$.w, where $(w, 2.5 .13 .17)=1$. It now follows from $(3.1 a)$ and $(3.1 b)$ that

$$
n=2^{7} \cdot 5^{2} \cdot 17^{c} \cdot 13^{d} \cdot w,
$$

and

$$
2^{6} \cdot 5 \cdot 17^{c-1} \cdot 13^{d-1} \cdot w=\sigma^{* *}\left(17^{c}\right) \cdot \sigma^{* *}\left(13^{d}\right) \cdot \sigma^{* *}(w)
$$

where

$$
w \text { has not more than four odd prime factors and }(w, 2.5 .13 .17)=1 \text {. }
$$

The rest of the proof of (b) of Theorem 3.1 depends on the following lemmas:

Lemma 3.1. Assume that $n$ given in (3.3a) is a bi-unitary triperfect number.

(i) If $c=1$, then $3^{2} \| n$.

(ii) If $c=d=1$, then $n=44553600=2^{7} \cdot 3^{2} \cdot 5^{2} \cdot 7 \cdot 13 \cdot 17$.

Proof. (i) Since $\sigma^{* *}(17)=18=2.3^{2}$, taking $c=1$ in $(3.3 b)$, we obtain

$$
2^{5} \cdot 5 \cdot 13^{d-1} \cdot w=3^{2} \cdot \sigma^{* *}\left(13^{d}\right) \cdot \sigma^{* *}(w) .
$$

Hence $3^{2} \mid w$ so that $w=3^{e} . w^{\prime}$, where $e \geq 2$ and $\left(w^{\prime}, 2.3 .5 .13 .17\right)=1$. From $(3.3 a)$ and $(3.3 d)$, we have

$$
n=2^{7} \cdot 5^{2} \cdot 17 \cdot 13^{d} \cdot 3^{e} \cdot w^{\prime}, \quad(e \geq 2)
$$

and

$$
2^{5} \cdot 5 \cdot 13^{d-1} \cdot 3^{e-2} \cdot w^{\prime}=\sigma^{* *}\left(13^{d}\right) \cdot \sigma^{* *}\left(3^{e}\right) \cdot \sigma^{* *}\left(w^{\prime}\right)
$$

where

$$
w^{\prime} \text { has not more than three odd prime factors and }\left(w^{\prime}, 2.3 .5 .13 .17\right)=1 .
$$


When $e \geq 3$, by Lemma $2.1, \frac{\sigma^{* *}\left(3^{e}\right)}{3^{e}} \geq \frac{112}{81}$. Using this from $(3.4 a)$, we obtain

$$
3=\frac{\sigma^{* *}(n)}{n} \geq \frac{255}{128} \cdot \frac{26}{25} \cdot \frac{18}{17} \cdot \frac{112}{81}=3.033>3
$$

a contradiction.

Hence $e \geq 3$ is not possible. Since $e \geq 2$, we must have $e=2$. Thus $3^{2} \| n$. This proves (i). Note 3.1. Taking $e=2$, in $(3.4 a)$ and $(3.4 b)$, we obtain

$$
n=2^{7} \cdot 5^{2} \cdot 17 \cdot 13^{d} \cdot 3^{2} \cdot w^{\prime}
$$

and

$$
2^{4} \cdot 13^{d-1} \cdot w^{\prime}=\sigma^{* *}\left(13^{d}\right) \cdot \sigma^{* *}\left(w^{\prime}\right)
$$

where

$$
w^{\prime} \text { has not more than three odd prime factors and }\left(w^{\prime \prime}, 2.3 .5 .13 .17\right)=1 \text {. }
$$

(ii) From (i), $c=1$ implies $e=2$. Taking $d=1$ in $(3.5 b)$, since $\sigma^{* *}(13)=14=2.7$, we find that $7 \mid w^{\prime}$ so that $w^{\prime}=7^{f} . w^{\prime \prime}$. Using these results in $(3.5 b)$ and $(3.4 a)(d=1)$, we obtain

$$
n=2^{7} \cdot 5^{2} \cdot 17 \cdot 13 \cdot 3^{2} \cdot 7^{f} \cdot w^{\prime \prime}
$$

and

$$
2^{3} \cdot 7^{f-1} \cdot w^{\prime \prime}=\sigma^{* *}\left(7^{f}\right) \cdot \sigma^{* *}\left(w^{\prime \prime}\right)
$$

where

$$
w^{\prime \prime} \text { has not more than two odd prime factors and }\left(w^{\prime \prime}, 2.3 .5 .7 .13 .17\right)=1 \text {. }
$$

Let $f=1$. From $(3.6 b)$, we have $w^{\prime \prime}=1$. Hence form $(3.5 a), n=7.5^{2} \cdot 17 \cdot 13.3^{2} .7=$ 44553600 .

If $f=2$, then since $\sigma^{* *}\left(7^{2}\right)=50=2.5^{2}$, from $(3.6 b)(f=2)$, it follows that $5 \mid w^{\prime \prime}$. But $w^{\prime \prime}$ is prime to 5 .

We may assume that $f \geq 3$. From Lemma 2.1, $\frac{\sigma^{* *}\left(7^{f}\right)}{7^{f}} \geq \frac{2752}{2401}$. Hence from $(3.6 a)$, we obtain

$$
3=\frac{\sigma^{* *}(n)}{n} \geq \frac{255}{128} \cdot \frac{26}{25} \cdot \frac{18}{17} \cdot \frac{14}{13} \cdot \frac{10}{9} \cdot \frac{2752}{2401}=3.008746356>3,
$$

a contradiction. This proves (ii) and the proof of Lemma 3.1 is complete.

Note 3.2. If $c=1$ and $d=2$, since $\sigma^{* *}\left(13^{2}\right)=170$, it follows from $(3.5 b)(d=2)$ that 17 divides its left-hand side. But this is not possible. Hence we may assume that $d \geq 3$ (the case $c=d=1$ is settled in (ii) of Lemma 3.1).

Lemma 3.2. Let $n$ be as given in (3.5a) with $d \geq 3$. If $n$ is a bi-unitary triperfect number then $7 \nmid n$. 
Proof. By our assumption, $(3.5 b)$ and $(3.5 c)$ are valid. Suppose that $7 \mid n$. We arrive at a contradiction as follows.

From (3.5a), $7 \mid w^{\prime}$. Let $w^{\prime}=7^{f} . w^{\prime \prime}$; using this in (3.5a) and (3.5b), we get

$$
n=2^{7} \cdot 5^{2} \cdot 17 \cdot 13^{d} \cdot 3^{2} \cdot 7^{f} \cdot w^{\prime \prime} \quad(d \geq 3)
$$

and

$$
2^{4} \cdot 13^{d-1} \cdot 7^{f} \cdot w^{\prime \prime}=\sigma^{* *}\left(13^{d}\right) \cdot \sigma^{* *}\left(7^{f}\right) \cdot \sigma^{* *}\left(w^{\prime \prime}\right)
$$

where

$$
w^{\prime \prime} \text { has not more than three odd prime factors and }\left(w^{\prime \prime}, 2.3 .5 .7 .13 .17\right)=1 \text {. }
$$

Since $d \geq 3$, by Lemma 2.1, $\frac{\sigma^{* *}\left(13^{d}\right)}{13^{d}} \geq \frac{30772}{28561}$; also, for $f \geq 3, \frac{\sigma^{* *}\left(7^{f}\right)}{7^{f}} \geq \frac{2752}{2401}$. From $(3.7 a)$, for $f \geq 3$, we have

$$
3=\frac{\sigma^{* *}(n)}{n} \geq \frac{255}{128} \cdot \frac{26}{25} \cdot \frac{18}{17} \cdot \frac{30772}{28561} \cdot \frac{10}{9} \cdot \frac{2752}{2401}=3.0110115835>3,
$$

a contradiction.

Hence $f=1$ or $f=2$.

Let $f=1$. From $(3.7 a)(f=1)$, we have

$$
3=\frac{\sigma^{* *}(n)}{n} \geq \frac{255}{128} \cdot \frac{26}{25} \cdot \frac{18}{17} \cdot \frac{30772}{28561} \cdot \frac{10}{9} \cdot \frac{8}{7}=3.00136598>3,
$$

a contradiction.

Let $f=2$. Since $\sigma^{* *}\left(7^{2}\right)=50$, taking $f=2$ in $(3.7 b)$, we find that $5 \mid w^{\prime \prime}$, which is false. Thus $7 \nmid n$.

The proof of Lemma 3.2 is complete.

Lemma 3.3. Let $n$ be as given in (3.5a), and let $n$ be a bi-unitary triperfect number.

(a) Then $d$ can neither be odd nor $4 \mid d$.

(b) Let $d=2 k$ where $k$ is odd and $k \geq 3$. We have

$$
\sigma^{* *}\left(13^{d}\right)=\left(\frac{13^{k}-1}{12}\right) \cdot\left(13^{k+1}+1\right) .
$$

Here,
(i) $\frac{13^{k}-1}{12}$ is divisible by a prime $p^{\prime} \mid w^{\prime}$ and $p^{\prime}>61$,
(ii) $\frac{13^{k+1}+1}{2}$ is divisible by a prime $q^{\prime} \mid w^{\prime}$ and $q^{\prime}>61$.

Proof. We assume that $n$ is a bi-unitary triperfect number. Thus (3.5b) and (3.5c) are valid.

(a) If $d$ is odd or $4 \mid d$, then $7 \mid \sigma^{* *}\left(13^{d}\right)$. It follows from $(3.5 b)$ that $7\left|w^{\prime}\right| n$. By Lemma 3.2, $7 \nmid n$. This proves (a).

(b) Let $d=2 k$, where $k$ is odd. Since $d \geq 3$, we have $k \geq 3$. 
(i) Let

$$
S_{13}^{\prime}=\left\{p \mid 13^{k}-1: p \in[3,61]-\{3,61\} \text { and } \operatorname{ord}_{p} 13 \text { is odd }\right\}
$$

Let us replace the interval $[3,293]$ by $[3,61]$ in Lemma 2.5 (a). Then it follows quickly that (i) is true when $S_{13}^{\prime}$ is non-empty.

We may assume that $S_{13}^{\prime}$ is empty. Since $p \nmid 13^{k}-1$ if $\operatorname{ord}_{p} 13$ is even, it follows that $13^{k}-1$ is not divisible by any prime $p \in[3,61]$ except for possibly $p=3,61$; but from $(3.5 b), \frac{13^{k}-1}{12} \mid \sigma^{* *}\left(13^{d}\right)$ is not divisible by 3 . We may note that $9 \mid 13^{k}-1 \Longleftrightarrow k=3 u \Longleftrightarrow$ $61 \mid 13^{k}-1$. Since $13^{k}-1$ is not divisible by 3 , it is not divisible by 61 either. It now follows that $\frac{13^{k}-1}{12}$ is not divisible by any prime in $[3,61]$. Since $\frac{13^{k}-1}{12}$ is odd and $>1$, we can find an odd prime $p^{\prime} \mid \frac{13^{k}-1}{12}$. Clearly, $p^{\prime}>61$ and from (3.5b), $p^{\prime} \mid w^{\prime}$. This proves (i).

(ii) Let

$$
T_{13}^{\prime}=\left\{q \mid 13^{k+1}+1: q \in[3,61]-\{5,17\} \text { and } s=\frac{1}{2} \operatorname{ord}_{p} 13 \text { is even }\right\} .
$$

Replacing the interval $[3,293]$ in Lemma 2.5 (b) by [3,61], we infer that (ii) holds if $T_{13}^{\prime}$ is non-empty.

Suppose that $T_{13}^{\prime}$ is empty. Since $q \nmid 13^{k+1}+1$ if $s=\frac{1}{2} \operatorname{ord}_{p} 13$ is odd, it follows that $\frac{13^{k+1}+1}{2}$ is not divisible by any prime $q \in[3,61]$ except for possibly $q=5$ or $q=17$.

It may be noted that $5\left|13^{k+1}+1 \Longleftrightarrow k+1=2 u \Longleftrightarrow 17\right| 13^{k+1}+1$. From $(3.5 b), 5$ is not a factor of its left-hand side and so $5 \nmid 13^{k+1}+1 \mid \sigma^{* *}\left(13^{d}\right)$. Hence $17 \nmid \frac{13^{k+1}+1}{2}$. Thus $\frac{13^{k+1}+1}{2}$ is odd, $>1$ and not divisible by any prime in $[3,61]$. Let $q^{\prime} \mid \frac{13^{k+1}+1}{2}$. Then $q^{\prime}>61$ and $q^{\prime} \mid w^{\prime}$ by $(3.5 b)$. This proves (ii).

The proof of Lemma 3.3 is complete.

Lemma 3.4. Let $n$ be as given in (3.5a) with $d \geq 3$. Then $n$ cannot a bi-unitary triperfect number.

Proof. On the contrary, assume that $n$ is a bi-unitary triperfect number.

By Lemma 3.2, $7 \nmid n$. Hence from (3.5a) each prime factor of $w^{\prime}$ can be assumed to be $\geq 11$. By Lemma 3.3, $w^{\prime}$ is divisible by two distinct odd prime factors $p^{\prime}>61$ and $q^{\prime}>61$. We may assume without loss of generality that $p^{\prime} \geq 67$ and $q^{\prime} \geq 71$. By $(3.5 c), w^{\prime}$ cannot have not more than three odd prime factors. If $y$ denotes a possible third prime factor of $w^{\prime}$ we may assume that $y \geq 11$ and $w^{\prime}=p^{\prime f} \cdot q^{\prime g} \cdot y^{h}$. From $(3.5 a)$, we have $n=2^{7} \cdot 5^{2} \cdot 17 \cdot 13^{d} \cdot 3^{2} \cdot p^{\prime f} \cdot q^{\prime g} \cdot y^{h}$. Hence

$$
3=\frac{\sigma^{* *}(n)}{n}<\frac{255}{128} \cdot \frac{26}{25} \cdot \frac{18}{17} \cdot \frac{13}{12} \cdot \frac{10}{9} \cdot \frac{67}{66} \cdot \frac{71}{70} \cdot \frac{11}{10}=2.990822173<3,
$$

a contradiction. This proves Lemma 3.4 .

Remark 3.1. Thus we have proved that when $b=2$, the case (i) $c=1, d=1$ yields the bi-unitary perfect number $n=44553600$. The cases (ii) $c=1, d=2$ and (iii) $c=1, d \geq 3$ lead to a contradiction. So when $b=2$ we may assume that $c \geq 2$. 
Remark 3.2. Let $b=2$ and $c \geq 2$. If $n=2^{7} \cdot 5^{2} \cdot 17^{c} \cdot 13^{d} . w$, where $(w, 2.5 .13 .17)=1,3^{e} \| n$ and $n$ is a bi-unitary triperfect number, then taking $w=3^{e} . w^{\prime}$ in $(3.3 a)$ and $(3.3 b)$, we obtain the following:

$$
n=2^{7} \cdot 5^{2} \cdot 17^{c} \cdot 13^{d} \cdot 3^{e} \cdot w^{\prime}, \quad(c \geq 2),
$$

and

$$
2^{6} \cdot 5 \cdot 17^{c-1} \cdot 13^{d-1} \cdot 3^{e} \cdot w^{\prime}=\sigma^{* *}\left(17^{c}\right) \cdot \sigma^{* *}\left(13^{d}\right) \cdot \sigma^{* *}\left(3^{e}\right) \cdot \sigma^{* *}\left(w^{\prime}\right)
$$

where

$$
w^{\prime} \text { has not more than three odd prime factors and }\left(w^{\prime}, 2.3 .5 .13 .17\right)=1 \text {. }
$$

Lemma 3.5. Let $n=2^{7} \cdot 5^{2} \cdot 17^{c} \cdot 13^{d} \cdot 3^{e} \cdot w^{\prime}(c \geq 2)$ be as in $(3.8 a)$ and $\left(w^{\prime}, 2.3 .5 .13 .17\right)=1$. Then $n$ cannot be a bi-unitary triperfect number if $c \geq 3$ and $e \geq 3$.

Proof. Let $c \geq 3$ and $e \geq 3$. We assume that $n$ is a bi-unitary triperfect number and obtain a contradiction.

By Lemma 2.1, for $c \geq 3, \frac{\sigma^{* *}\left(17^{c}\right)}{17^{c}} \geq \frac{88452}{83521}$ and for $e \geq 3, \frac{\sigma^{* *}\left(3^{e}\right)}{3^{e}} \geq \frac{112}{81}$. Hence from $(3.8 a)$ for $c \geq 3$ and $e \geq 3$,

$$
3=\frac{\sigma^{* *}(n)}{n} \geq \frac{255}{128} \cdot \frac{26}{25} \cdot \frac{88452}{83521} \cdot \frac{112}{81}=3.033950743>3,
$$

a contradiction. This proves Lemma 3.5.

Remark 3.3. In order to prove that $n$ given in $(3.8 a)$ is not a bi-unitary triperfect number, in view of Lemma 3.5, it remains to examine the cases (I) $c=2, e \geq 3$, (II) $c \geq 3, e=1$ or 2 , (III) $c=2$, $e=1$ or 2 .

In the following Lemmas 3.6 to 3.8, we deal with the three cases mentioned in Remark 3.3.

Lemma 3.6. The number $n$ given in $(3.8 a)$ with $c=2$ and $e \geq 3$ cannot be a bi-unitary triperfect number.

Proof. Assume that $n$ in $(3.8 a)$ with $c=2$ and $e \geq 3$ is a bi-unitary triperfect number. We can use $(3.8 b)$ and $(3.8 c)$. Since $\sigma^{* *}\left(17^{2}\right)=290=2.5 .29$, taking $c=2$ in $(3.8 b)$, we get after simplification,

$$
2^{5} \cdot 17 \cdot 13^{d-1} \cdot 3^{e} \cdot w^{\prime}=29 \cdot \sigma^{* *}\left(13^{d}\right) \cdot \sigma^{* *}\left(3^{e}\right) \cdot \sigma^{* *}\left(w^{\prime}\right) .
$$

From $(3.8 d)$, it follows that $29 \mid w^{\prime}$. Let $w^{\prime}=29^{f} . w^{\prime \prime}$. From $(3.8 a)$ and $(3.8 d)$, we have

$$
n=2^{7} \cdot 5^{2} \cdot 17^{2} \cdot 13^{d} \cdot 3^{e} \cdot 29^{f} \cdot w^{\prime \prime},
$$

and

$$
2^{5} \cdot 17 \cdot 13^{d-1} \cdot 3^{e} \cdot 29^{f-1} \cdot w^{\prime \prime}=\sigma^{* *}\left(13^{d}\right) \cdot \sigma^{* *}\left(3^{e}\right) \cdot \sigma^{* *}\left(29^{f}\right) \cdot \sigma^{* *}\left(w^{\prime \prime}\right),
$$

where

$$
w^{\prime \prime} \text { has not more than two odd prime factors and }\left(w^{\prime \prime}, 2.3 .5 .13 .17 .29\right)=1 \text {. }
$$


By Lemma 2.1, for $d \geq 3, \frac{\sigma^{* *}\left(13^{d}\right)}{13^{d}} \geq \frac{30772}{28561}$; also, for $e \geq 3, \frac{\sigma^{* *}\left(3^{e}\right)}{3^{e}} \geq \frac{112}{81}$. Hence from $(3.9 a)$, for $d \geq 3$, we have

$$
3=\frac{\sigma^{* *}(n)}{n} \geq \frac{255}{128} \cdot \frac{26}{25} \cdot \frac{290}{289} \cdot \frac{30772}{28561} \cdot \frac{112}{81}=3.097269697>3,
$$

a contradiction.

Hence $d=1$ or $d=2$.

When $d=1$, again from $(3.9 a)(d=1)$, we have

$$
3=\frac{\sigma^{* *}(n)}{n} \geq \frac{255}{128} \cdot \frac{26}{25} \cdot \frac{290}{289} \cdot \frac{14}{13} \cdot \frac{112}{81}=3.095860566>3,
$$

a contradiction.

Let $d=2$. Since $\sigma^{* *}\left(13^{2}\right)=170=2.5 .17$, taking $d=2$ in $(3.9 b)$, we find that 5 is a factor of the left-hand side of $(3.9 b)$. This is false. This completes the proof of Lemma 3.6.

Lemma 3.7. The number $n$ given in (3.8a) with $c \geq 3$ and $e=1$ or 2 cannot be a bi-unitary triperfect number.

Proof. We assume that $n$ is a bi-unitary triperfect number and obtain a contradiction. Since $c \geq 3$, by Lemma 2.1, $\frac{\sigma^{* *}\left(17^{c}\right)}{17^{c}} \geq \frac{88452}{83521}$. Also, for $d \geq 3, \frac{\sigma^{* *}\left(13^{d}\right)}{13^{d}} \geq \frac{30772}{28561}$.

Let $e=1$. Hence from $(3.8 a)(e=1)$, for $d \geq 3$,

$$
3=\frac{\sigma^{* *}(n)}{n} \geq \frac{255}{128} \cdot \frac{26}{25} \cdot \frac{88452}{83521} \cdot \frac{30772}{28561} \cdot \frac{4}{3}=3.152075221>3,
$$

a contradiction.

Hence $d=1$ or $d=2$.

If $d=1$, from $(3.8 a)(d=1, e=1)$, we have

$$
3=\frac{\sigma^{* *}(n)}{n} \geq \frac{255}{128} \cdot \frac{26}{25} \cdot \frac{14}{13} \cdot \frac{30772}{28561} \cdot \frac{4}{3}=3.150614156>3,
$$

a contradiction.

Let $d=2($ already $e=1)$. We have from $(3.8 a)$

$$
n=2^{7} \cdot 5^{2} \cdot 17^{c} \cdot 13^{2} \cdot 3 \cdot w^{\prime}, \quad(c \geq 3)
$$

and from $(3.8 b)(d=2, e=1)$, since $\sigma^{* *}\left(13^{2}\right)=2.5 .17$, we get after simplification

$$
2^{3} \cdot 17^{c-2} \cdot 13 \cdot 3 \cdot w^{\prime}=\sigma^{* *}\left(17^{c}\right) \cdot \sigma^{* *}\left(w^{\prime}\right)
$$

$w^{\prime}$ has not more than two odd prime factors and $\left(w^{\prime}, 2.3 .5 .13 .17\right)=1$.

Let $c$ be odd. Then $17^{2}-1=288 \mid 17^{c+1}-1$. Hence $9 \mid \frac{17^{c+1}-1}{16}=\sigma^{* *}\left(17^{c}\right)$. From $(3.10 b)$, it follows that $3 \mid w^{\prime}$. This is not possible.

Let $c$ be even, say $c=2 k$. We have

$$
\sigma^{* *}\left(17^{c}\right)=\left(\frac{17^{k}-1}{16}\right) \cdot\left(17^{k+1}+1\right)
$$

If $k$ is even, then $9\left|\frac{17^{k}-1}{16}\right| \sigma^{* *}\left(17^{c}\right)$. This leads to a contradiction as before. 
We may assume that $c=2 k$ and $k$ is odd. Since $c \geq 3$, we have $k \geq 3$. We prove that:

(I) $\frac{17^{k}-1}{16}$ is divisible by an odd prime $p^{\prime} \mid w^{\prime}$ and $p^{\prime}>127$,

(II) $\frac{17^{k+1}+1}{2}$ is divisible by an odd prime $q^{\prime} \mid w^{\prime}$ and $q^{\prime}>127$.

- Proof of (I). Let

$$
S_{17}^{\prime}=\left\{p \mid 17^{k}-1: p \in[3,127] \text { and } \operatorname{ord}_{p} 17 \text { is odd }\right\}
$$

By Lemma 2.6(a), if $S_{17}^{\prime}$ is non-empty, then (I) holds. Suppose that $S_{17}^{\prime}$ is empty. Since $p \nmid 17^{k}-1$ if $\operatorname{ord}_{p} 17$ is even (and $k$ is odd), it follows that $\frac{17^{k}-1}{16}$ is not divisible by any prime in $[3,127]$. Since $\frac{17^{k}-1}{16}$ is odd, $>1$, it must be divisible by an odd prime $p^{\prime}$ and clearly $p^{\prime}>127$. Also, from (3.10b), $p^{\prime} \mid w^{\prime}$. This proves (I).

- Proof of (II). Let

$$
T_{17}^{\prime}=\left\{q \mid 17^{k+1}+1: q \in[3,127]-\{5,29\} \text { and } s=\frac{1}{2} \operatorname{ord}_{q} 17 \text { is even }\right\} .
$$

By Lemma 2.6(b), if $T_{17}^{\prime}$ is non-empty, (II) holds. So we may assume that $T_{17}^{\prime}$ is empty. Since $s=\frac{1}{2} \operatorname{ord}_{q} 17$ is not even implies that $q \nmid 17^{k+1}+1$ it follows that $\frac{17^{k+1}+1}{2}$ is divisible by none of the primes in $[3,127]$ except for possibly 5 or 29 .

We may note that $5\left|17^{k+1}+1 \Longleftrightarrow k+1=2 u \Longleftrightarrow 29\right| 17^{k+1}+1$. Let $5 \mid 17^{k+1}+1$. Since $17^{k+1}+1 \mid \sigma^{* *}\left(17^{c}\right)$, it follows from $(3.10 b)$ that 5 is a factor of the left-hand side of it. But this is false. Hence $5 \nmid 17^{k+1}+1$ and hence $29 \nmid 17^{k+1}+1$. Thus $\frac{17^{k+1}+1}{2}$ is not divisible by any prime in $[3,127]$. Since $\frac{17^{k+1}+1}{2}$ is $>1$ and odd, we can find an odd prime $q^{\prime} \mid \frac{17^{k+1}+1}{2}$. Clearly $q^{\prime}>127$ and $q^{\prime} \mid w^{\prime}$ from (3.10b). This proves (II).

Since $\frac{17^{k}-1}{16}$ and $\frac{17^{k+1}+1}{2}$ are relatively prime, we have $p^{\prime} \neq q^{\prime}$. We may assume that $p^{\prime} \geq 131$ and $q^{\prime} \geq 137$. By $(3.10 c), w^{\prime}=\left(p^{\prime}\right)^{f} \cdot\left(q^{\prime}\right)^{g}$. Hence from $(3.10 a), n=2^{7} \cdot 5^{2} \cdot 17^{c} .13^{2} .3 .\left(p^{\prime}\right)^{f} \cdot\left(q^{\prime}\right)^{g}$ and so

$$
3=\frac{\sigma^{* *}(n)}{n}<\frac{255}{128} \cdot \frac{26}{25} \cdot \frac{17}{16} \cdot \frac{170}{169} \cdot \frac{4}{3} \cdot \frac{131}{130} \cdot \frac{137}{136}=2.997112495<3,
$$

a contradiction. This proves that $e=1$ is not possible.

Let $e=2$. Since $\sigma^{* *}\left(3^{2}\right)=10=2.5$, taking $e=2$ in $(3.8 a)$ and $(3.8 b)$, we obtain

$$
n=2^{7} \cdot 5^{2} \cdot 17^{c} \cdot 13^{d} \cdot 3^{2} \cdot w^{\prime}, \quad(c \geq 3)
$$

and

$$
2^{5} \cdot 17^{c-1} \cdot 13^{d-1} \cdot 3^{2} \cdot w^{\prime}=\sigma^{* *}\left(17^{c}\right) \cdot \sigma^{* *}\left(13^{d}\right) \cdot \sigma^{* *}\left(w^{\prime}\right)
$$

where

$w^{\prime}$ has not more than three odd prime factors and $\left(w^{\prime}, 2.3 .5 .13 .17\right)=1$. 
Since $\sigma^{* *}\left(13^{2}\right)=170=2.5 .17$, taking $d=2$ in $(3.11 b)$, we see that 5 is a factor of its left-hand side. But this is not so. Hence we may assume that $d \neq 2$.

Let $d=1$. Since $\sigma^{* *}(13)=14=2.7$, taking $d=1$ in $(3.11 b)$, we see that $7 \mid w^{\prime}$. Let $w^{\prime}=7^{f} . w^{\prime \prime}$. From $(3.11 a)$ and $(3.11 b)$, we have

$$
n=2^{7} \cdot 5^{2} \cdot 17^{c} \cdot 13^{d} \cdot 3^{2} \cdot 7^{f} \cdot w^{\prime \prime}, \quad(c \geq 3)
$$

and

$$
2^{4} \cdot 17^{c-1} \cdot 3^{2} \cdot 7^{f-1} \cdot w^{\prime \prime}=\sigma^{* *}\left(17^{c}\right) \cdot \sigma^{* *}\left(7^{f}\right) \cdot \sigma^{* *}\left(w^{\prime \prime}\right)
$$

where

$$
w^{\prime \prime} \text { has not more than two odd prime factors and }\left(w^{\prime}, 2.3 .5 .7 .13 .17\right)=1 \text {. }
$$

By Lemma 2.1, for $f \geq 3 \frac{\sigma^{* *}\left(7^{f}\right)}{7^{f}} \geq \frac{2752}{2401}$ and since $c \geq 3 \frac{\sigma^{* *}\left(17^{c}\right)}{\left.17^{c}\right)} \geq \frac{88452}{83521}$. Hence from $(3.12 a)$,

$$
3=\frac{\sigma^{* *}(n)}{n} \geq \frac{255}{128} \cdot \frac{26}{25} \cdot \frac{88452}{83521} \cdot \frac{14}{13} \cdot \frac{10}{9} \cdot \frac{2752}{2401}=3.009358761>3,
$$

a contradiction.

Hence $f=1$ or $f=2$ when $d=1$.

If $f=1$, from $(3.12 a)(f=1)$, we have

$$
3=\frac{\sigma^{* *}(n)}{n} \geq \frac{255}{128} \cdot \frac{26}{25} \cdot \frac{88452}{83521} \cdot \frac{14}{13} \cdot \frac{10}{9} \cdot \frac{8}{7}=3.000610625>3,
$$

a contradiction.

Let $f=2$. Since $\sigma^{* *}\left(7^{2}\right)=50=2.5^{2}$, taking $f=2$ in $(3.12 b)$, we find that 5 is a factor of its left-hand side which is false. Thus $d=1$ is not admissible. Since $d \neq 2$, we may assume that $d \geq 3$.

Thus $c \geq 3, d \geq 3$ and $e=2$. The relevant equations are (3.11a) to (3.11c).

We now show that $7 \nmid n$ when $n$ is as given in (3.11a). On the contrary, assume that $7 \mid n$. Hence $7 \mid w^{\prime}$ and let $w^{\prime}=7^{f} . w^{\prime \prime}$. From $(3.11 a)$ and (3.11b), we get

$$
n=2^{7} \cdot 5^{2} \cdot 17^{c} \cdot 13^{d} \cdot 3^{2} \cdot 7^{f} \cdot w^{\prime \prime}, \quad(c \geq 3, d \geq 3)
$$

and

$$
2^{5} \cdot 17^{c-1} \cdot 13^{d-1} \cdot 3^{2} \cdot 7^{f} \cdot w^{\prime \prime}=\sigma^{* *}\left(17^{c}\right) \cdot \sigma^{* *}\left(13^{d}\right) \cdot \sigma^{* *}\left(7^{f}\right) \cdot \sigma^{* *}\left(w^{\prime \prime}\right)
$$

where

$$
w^{\prime \prime} \text { has not more than two odd prime factors and }\left(w^{\prime \prime}, 2.3 .5 .7 .13 .17\right)=1 \text {. }
$$

Since $c$ and $d$ are $\geq 3$, we have by Lemma 2.1, $\frac{\sigma^{* *}\left(17^{c}\right)}{17^{c}} \geq \frac{88452}{83521}$ and $\frac{\sigma^{* *}\left(13^{d}\right)}{\left.13^{d}\right)} \geq \frac{30772}{28561}$. Also, for $f \geq 3$, we have $\frac{\sigma^{* *}\left(7^{f}\right)}{7^{f}} \geq \frac{2752}{2401}$. Using these results, from (3.13a), we obtain for $f \geq 3$,

$$
3=\frac{\sigma^{* *}(n)}{n} \geq \frac{255}{128} \cdot \frac{26}{25} \cdot \frac{88452}{83521} \cdot \frac{30772}{28561} \cdot \frac{10}{9} \cdot \frac{2752}{2401}=3.010728519>3,
$$

a contradiction. Hence $f=1$ or $f=2$. 
If $f=1$, from $(3.13 a)(f=1)$,

$$
3=\frac{\sigma^{* *}(n)}{n} \geq \frac{255}{128} \cdot \frac{26}{25} \cdot \frac{88452}{83521} \cdot \frac{30772}{28561} \cdot \frac{10}{9} \cdot \frac{8}{7}=3.001976401>3,
$$

a contradiction.

Let $f=2$. Since $\sigma^{* *}\left(7^{2}\right)=50=2.5^{2}$, taking $f=2$ in $(3.13 b)$, we see that 5 is a factor of its left-hand side and this is false. Hence $7 \nmid n$.

We return to the equations $(3.11 a)-(3.11 c)$ in which $7 \nmid n$ or equivalently $7 \nmid w^{\prime}$. We can assume that each prime factor of $w^{\prime}$ in $(3.11 a)-(3.11 c)$ is at least 11.

We examine the factors of $\sigma^{* *}\left(13^{d}\right)$ and obtain a contradiction when $e=2$.

If $d$ is odd or $4 \mid d$, then $\sigma^{* *}\left(13^{d}\right)$ is divisible by 7 . From $(3.11 b)$ it follows that $7\left|w^{\prime}\right| n$. But $7 \nmid n$.

Hence we may assume that $d=2 k$, where $k$ is odd and $k \geq 3$, since $d \geq 3$. We have

$$
\sigma^{* *}\left(13^{d}\right)=\left(\frac{13^{k}-1}{12}\right) \cdot\left(13^{k+1}+1\right) \text {. }
$$

We prove that:

(III) $\frac{13^{k}-1}{12}$ is divisible by a prime $p^{\prime} \mid w^{\prime}$ and $p^{\prime}>127$,

(IV) $\frac{13^{k+1}+1}{2}$ is divisible by a prime $q^{\prime} \mid w^{\prime}$ and $q^{\prime}>127$.

- Proof of (III). Let

$$
S_{13}^{\prime}=\left\{p \mid 13^{k}-1: p \in[3,127]-\{3,61\} \text { and } \operatorname{ord}_{p} 13 \text { is odd }\right\} .
$$

If we replace the interval $[3,293]$ in Lemma 2.5(a), by [3, 127], it follows that (III) holds if $S_{13}^{\prime}$ is non-empty.

Suppose that $S_{13}^{\prime}$ is empty. Since $p \nmid 13^{k}-1$ if $\operatorname{ord}_{p} 13$ is even, it follows that $\frac{13^{k}-1}{12}$ is not divisible by any prime in $[3,127]$ except for possibly 3 and 61.

Clearly $3 \mid 13^{k}-1$. We show that $27 \nmid 13^{k}-1$. On the contrary, suppose that $27 \mid 13^{k}-1$. This is equivalent to $9 \mid k$. Hence $13^{9}-1 \mid 13^{k}-1$. Also, $13^{9}-1=2^{2} \cdot 3^{3} .61 .1609669$. Hence 61 and 1609669 are factors of $w^{\prime}$ and by $(3.11 c), w^{\prime}=61^{f} .(1609669)^{g} \cdot w^{\prime \prime}$, where $w^{\prime \prime}=1$ or $w^{\prime \prime}=p^{\alpha}$ where $p \geq 11$. Hence $\sigma^{* *}\left(w^{\prime \prime}\right) / w^{\prime \prime}<11 / 10$. From $(3.11 a)$,

$$
n=2^{7} \cdot 5^{2} \cdot 17^{c} \cdot 13^{d} \cdot 3^{2} \cdot 61^{f} \cdot(1609669)^{g} \cdot w^{\prime \prime}
$$

so that

$$
3=\frac{\sigma^{* *}(n)}{n}<\frac{255}{128} \cdot \frac{26}{25} \cdot \frac{17}{16} \cdot \frac{13}{12} \cdot \frac{10}{9} \cdot \frac{61}{60} \cdot \frac{1609669}{1609668} \cdot \frac{11}{10}=2.963354615<3,
$$

a contradiction. Hence $27 \nmid 13^{k}-1$.

We may note that $9\left|13^{k}-1 \Longleftrightarrow 3\right| k \Longleftrightarrow 61 \mid 13^{k}-1$.

Assume that $9 \nmid 13^{k}-1$. Then $61 \nmid 13^{k}-1$. Thus $\frac{13^{k}-1}{12}>1$, odd and not divisible by 3 and 61 ; and so not divisible by any prime in $[3,127]$. If $p^{\prime} \mid \frac{13^{k}-1}{12}$, then $p^{\prime}>127$ and $p^{\prime} \mid w^{\prime}$ by $(3.11 b)$. This proves (III) in this case. 
Suppose that $9 \mid 13^{k}-1$. Then $61 \mid 13^{k}-1$. Also, $\frac{13^{k}-1}{36}>1$, odd and not divisible by 3 but divisible by 61 . We show that $\frac{13^{k}-1}{36}$ must be divisible by an odd prime other than 61 . If this is not so let $\frac{13^{k}-1}{36}=61^{\alpha}$, where $\alpha$ is a positive integer. If $\alpha \geq 2$, then $61^{2} \mid 13^{k}-1$. This holds if and only if $183 \mid k$. Hence, $367\left|13^{183}-1\right| 13^{k}-1$. Hence, $367 \mid \frac{13^{k}-1}{36}=61^{\alpha}$. This is not possible and so $\alpha=1$. Thus $\frac{13^{k}-1}{36}=61$ or $k=3$ or $d=6$.

We now show that $d=6$ is not admissible. We have $\sigma^{* *}\left(13^{6}\right)=2.3 .61 .14281$. Taking $d=6$ in $(3.11 b)$, we see that $w^{\prime}$ is divisible by 61 and 14281 . From $(3.11 c), w^{\prime}=$ $61^{f} .(14281)^{g} . w^{\prime \prime}$, where $w^{\prime \prime}=1$ or $p^{\alpha}$, where $p \geq 11$. Hence

$$
n=2^{7} \cdot 5^{2} \cdot 17^{c} \cdot 13^{6} \cdot 3^{2} \cdot 61^{f} \cdot(14281)^{g} \cdot w^{\prime \prime}
$$

so that

$$
3=\frac{\sigma^{* *}(n)}{n}<\frac{255}{128} \cdot \frac{26}{25} \cdot \frac{17}{16} \cdot \frac{13}{12} \cdot \frac{10}{9} \cdot \frac{61}{60} \cdot \frac{14281}{14280} \cdot \frac{11}{10}=2.963560292<3,
$$

a contradiction.

It now follows that $\frac{13^{k}-1}{36}$ is not divisible by 61 alone. Let $p^{\prime} \mid \frac{13^{k}-1}{36}$ and $p^{\prime} \neq 61$. It follows that $p^{\prime}>127$ and from (3.11b), $p^{\prime} \mid w^{\prime}$. This proves (III).

- $\underline{\text { Proof of (IV). Let }}$

$$
T_{13}^{\prime}=\left\{q \mid 13^{k+1}+1: q \in[3,127]-\{5,17\} \text { and } s=\frac{1}{2} \operatorname{ord}_{q} 13 \text { is even }\right\} .
$$

By Lemma 2.5 (b), (IV) holds if $T_{13}^{\prime}$ is non-empty. We may assume that $T_{13}^{\prime}$ is empty. Since $q \nmid 13^{k+1}+1$ if $s=\frac{1}{2} \operatorname{ord}_{q} 13$ is not even, it follows that $\frac{13^{k+1}+1}{2}$ is not divisible by any prime in $[3,127]$ except for possibly 5 and 17.

If $5 \mid 13^{k+1}+1$, then $5 \mid \sigma^{* *}\left(13^{d}\right)$ and from $(3.11 b)$, it follows that 5 divides its left-hand side. This is false. Hence $5 \nmid 13^{k+1}+1$. Since $5 \nmid 13^{k+1}+1 \Longleftrightarrow k+1=2 u \Longleftrightarrow 17 \nmid 13^{k+1}+1$, we conclude that $17 \nmid 13^{k+1}+1$.

Thus $\frac{13^{k+1}+1}{2}>1$ and is odd, and it is not divisible by any prime in $[3,127]$. Let $q^{\prime} \mid \frac{13^{k+1}+1}{2}$. Then $q^{\prime}>127$ and $q^{\prime} \mid w^{\prime}$ from $(3.11 b)$.

This proves (IV).

From (III), (IV) and (3.11c), $w^{\prime}=\left(p^{\prime}\right)^{f} \cdot\left(q^{\prime}\right)^{g} \cdot t^{h}$, where $t$ is the possible third prime factor of $w^{\prime}$ and $t \geq 11$. We can assume that $p^{\prime} \geq 131$ and $q^{\prime} \geq 137$.

From $(3.11 a), n=2^{7} \cdot 5^{2} \cdot 17^{c} \cdot 13^{d} \cdot 3^{2} \cdot\left(p^{\prime}\right)^{f} \cdot\left(q^{\prime}\right)^{d} \cdot t^{h}$, and so

$$
3=\frac{\sigma^{* *}(n)}{n}<\frac{255}{128} \cdot \frac{26}{25} \cdot \frac{17}{16} \cdot \frac{13}{12} \cdot \frac{10}{9} \cdot \frac{131}{130} \cdot \frac{137}{136} \cdot \frac{11}{10}=2.958791572<3,
$$

a contradiction. This completes the case $e=2$ and the proof of Lemma 3.7. 
Lemma 3.8. The number $n$ given in (3.8a) with $c=2$ and $e=1$ or 2 cannot be a bi-unitary triperfect number.

Proof. We assume that $n$ given in (3.8) is a bi-unitary triperfect number.

Let $c=2$ and $e=1$. Since $c=2$, we can use the equations (3.9a) to (3.9c). Taking $e=1$ in $(3.9 a)$ and $(3.9 b)$, we get after simplification

$$
n=2^{7} \cdot 5^{2} \cdot 17^{2} \cdot 13^{d} \cdot 3 \cdot 29^{f} \cdot w^{\prime \prime},
$$

and

$$
2^{3} \cdot 17 \cdot 13^{d-1} \cdot 3 \cdot 29^{f-1} \cdot w^{\prime \prime}=\sigma^{* *}\left(13^{d}\right) \cdot \sigma^{* *}\left(29^{f}\right) \cdot \sigma^{* *}\left(w^{\prime \prime}\right),
$$

where

$$
w^{\prime \prime}=1 \text { or a prime power. }
$$

Let $d=1$. Since $\sigma^{* *}(13)=14=2.7$, taking $d=1$ in $(3.14 b)$, we see that $7 \mid w^{\prime \prime}$. Hence form (3.14c), $w^{\prime \prime}=7^{g}$. From $(3.14 a)$ and $(3.14 b)$ we have

$$
n=2^{7} \cdot 5^{2} \cdot 17^{2} \cdot 13 \cdot 3 \cdot 29^{f} \cdot 7^{g},
$$

and

$$
2^{2} \cdot 17.3 .29^{f-1} \cdot 7^{g-1}=\sigma^{* *}\left(29^{f}\right) \cdot \sigma^{* *}\left(7^{g}\right) .
$$

Since $\sigma^{* *}(7)=8$, taking $g=1$ in $(3.15 b)$, we find that $2^{4}$ divides the right-hand side of it, whereas $2^{2}$ is a unitary divisor of its left-hand side. Hence $g=1$ is not possible.

Since $\sigma^{* *}\left(7^{2}\right)=50$, taking $g=2$ in $(3.15 b)$, we see that 5 divides its right-hand side but this is not true with respect to its left-hand side. Hence $g=2$ is also not possible.

We may assume that $g \geq 3$ so that $\frac{\sigma^{* *}\left(7^{g}\right)}{7^{g}} \geq \frac{2752}{2401}$. From $(3.14 a)$, we have

$$
3=\frac{\sigma^{* *}(n)}{n} \geq \frac{255}{128} \cdot \frac{26}{25} \cdot \frac{290}{289} \cdot \frac{14}{13} \cdot \frac{4}{3} \cdot \frac{2752}{2401}=3.421711542>3,
$$

a contradiction. Hence $d=1$ is not admissible.

Let $d=2$ in $(3.14 b)$. Since $\sigma^{* *}\left(13^{2}\right)=170=2.5 .17$, we find that 5 is a factor of its left-hand side. This cannot happen. Hence $d=2$ is not possible. We may assume that $d \geq 3$.

Let $f=1$ in $(3.14 b)$. Since $\sigma^{* *}(29)=30=2.3 .5$, we see that 5 is a factor of its left-hand side which is not true.

Let $f=2$. We have $\sigma^{* *}\left(29^{2}\right)=842=2.421$. From $(3.14 c), w^{\prime \prime}=(421)^{g}$. Hence from $(3.14 a)(f=2)$, we have

$$
n=2^{7} \cdot 5^{2} \cdot 17^{2} \cdot 13^{d} \cdot 3 \cdot 29^{2} \cdot(421)^{g},
$$

and from $(3.14 b)(f=2)$, we obtain

$$
2^{2} \cdot 17 \cdot 13^{d-1} \cdot 3 \cdot 29 \cdot(421)^{g-1}=\sigma^{* *}\left(13^{d}\right) \cdot \sigma^{* *}\left(29^{f}\right) \cdot \sigma^{* *}\left((421)^{g}\right) .
$$

We obtain a contradiction by examining the factors of $\sigma^{* *}\left(13^{d}\right)$.

If $d$ is odd or $4 \mid d$, then $7 \mid \sigma^{* *}\left(13^{d}\right)$. From $(3.16 b)$, we find that 7 divides the left-hand side of it. This cannot happen. 
We may assume that $d=2 k$ where $k$ is odd and $k \geq 3$, since $d \geq 3$. We have

$$
\sigma^{* *}\left(13^{d}\right)=\left(\frac{13^{k}-1}{12}\right) \cdot\left(13^{k+1}+1\right) \text {. }
$$

We prove that $\frac{13^{k}-1}{12}$ is not divisible by $2,3,5,17,29$, and 421 . This leads to a contradiction.

(i) Clearly $4 \mid 13^{k}-1$ but $8 \nmid 13^{k}-1$, since $k$ is odd. Hence $\frac{13^{k}-1}{12}$ is odd.

(ii) Clearly $3 \mid 13^{k}-1$. We note that $9\left|13^{k}-1 \Longleftrightarrow 3\right| k \Longleftrightarrow 61 \mid 13^{k}-1$. Suppose $9 \mid 13^{k}-1$. Hence $61 \mid 13^{k}-1$ and so $61\left|\frac{13^{k}-1}{12}\right| \sigma^{* *}\left(13^{d}\right)$. Thus 61 is a factor of the left-hand side of $(3.16 b)$. This is false. Hence $9 \nmid 13^{k}-1$. Hence $\frac{13^{k}-1}{12}$ is not divisible by 3 .

(iii) We have $17\left|13^{k}-1 \Longleftrightarrow 4\right| k ; 29\left|13^{k}-1 \Longleftrightarrow 14\right| k$; and $421\left|13^{k}-1 \Longleftrightarrow 20\right| k$. But $4,14,20$ cannot divide $k$ since $k$ is odd. Hence $\frac{13^{k}-1}{12}$ is not divisible by $17,29,421$.

From (i), (ii) and (iii), $\frac{13^{k}-1}{12}$ is not divisible by $2,3,17,29,421$ and trivially not divisible by 13. This cannot happen in view of $(3.16 b)$. Thus $f=2$ is not possible.

We may assume that $f \geq 3$. Since $d \geq 3$ and $f \geq 3$, by Lemma $2.1, \frac{\sigma^{* *}\left(13^{d}\right)}{13^{d}} \geq \frac{30772}{28561}$ and $\frac{\sigma^{* *}\left(29^{f}\right)}{29^{f}} \geq \frac{731700}{707281}$. From $(3.16 a)$, we obtain

$$
3=\frac{\sigma^{* *}(n)}{n} \geq \frac{255}{128} \cdot \frac{26}{25} \cdot \frac{290}{289} \cdot \frac{30772}{28561} \cdot \frac{4}{3} \cdot \frac{731700}{707281}=3.089767636>3,
$$

a contradiction.

This completes the case $c=2$ and $e=1$.

Let $c=2$ and $e=2$. Taking $e=2$ in $(3.9 b)$, we see that 5 is a factor of its right-hand side but it cannot divide its left-hand side. This completes the proof of Lemma 3.8.

We return to the equations $(3.3 a)-(3.3 c)$. In Lemmas 3.5 to 3.8 , we proved that if $3 \mid n, 5^{2} \| n$ and $17^{2} \mid n$, then $n$ cannot be a bi-unitary triperfect number. In what follows we will be prove that $n$ cannot be a bi-unitary triperfect number if $3 \nmid n, 5^{2} \| n$ and $17^{2} \mid n$.

Lemma 3.9. Let $n$ be as in (3.3a) with $c \geq 2$. Assume that $3 \nmid n$.

(a) If $7 \nmid n$, then $n$ is not a bi-unitary triperfect number.

(b) Assume that $n$ is a bi-unitary triperfect number. If $7 \mid n$, then $n$ is not divisible by $s$ where $s \in\{11,13,19,23\}$.

Proof. (a) Suppose $7 \nmid n$ and $n$ is a bi-unitary triperfect number. From the hypothesis, $n=2^{7} \cdot 5^{2} \cdot 17^{c} .13^{d} . w,(c \geq 2)$, where $w$ is prime to 2.5.7.13.17. Also, by $(3.3 c), w$ cannot have more than four odd prime factors. If $p_{1}, p_{2}, p_{3}$ and $p_{4}$ denote the four odd prime factors of $w$, we can assume that $p_{1} \geq 11, p_{2} \geq 19, p_{3} \geq 23$ and $p_{4} \geq 29$. We have from (3.3a), $n=2^{7} \cdot 5^{2} \cdot 17^{c} \cdot 13^{d} \cdot p_{1}^{e} \cdot p_{2}^{f} \cdot p_{3}^{g} \cdot p_{4}^{h}$, and by Lemma 2.1 ,

$$
3=\frac{\sigma^{* *}(n)}{n}<\frac{255}{128} \cdot \frac{26}{25} \cdot \frac{17}{16} \cdot \frac{13}{12} \cdot \frac{11}{10} \cdot \frac{19}{18} \cdot \frac{23}{22} \cdot \frac{29}{28}=2.998289044<3,
$$

a contradiction. Hence $n$ is not a bi-unitary triperfect number. This proves (a). 
(b) Assume that $7 \mid n$ so that $7 \mid w$. Let $w=7^{e} . w^{\prime}$. Using this in (3.3a) and (3.3b), we obtain

$$
n=2^{7} \cdot 5^{2} \cdot 17^{c} \cdot 13^{d} \cdot 7^{e} \cdot w^{\prime}, \quad(c \geq 2)
$$

and

$$
2^{6} \cdot 5 \cdot 17^{c-1} \cdot 13^{d-1} \cdot 7^{e} \cdot w^{\prime}=\sigma^{* *}\left(17^{c}\right) \cdot \sigma^{* *}\left(13^{d}\right) \cdot \sigma^{* *}\left(7^{e}\right) \cdot \sigma^{* *}\left(w^{\prime}\right)
$$

where

$w^{\prime}$ has not more than three odd prime factors and $\left(w^{\prime}, 2.3 .5 .7 .13 .17\right)=1$.

We next show that $11 \nmid n$. Suppose that $11 \mid n$. Hence $11 \mid w^{\prime}$. Let $w^{\prime}=11^{f} . w^{\prime \prime}$, where $\left(w^{\prime \prime}, 2.3 .5 .7 .11 .13 .17\right)=1$. From $(3.17 a)$ and $(3.17 b)$, we have

$$
n=2^{7} \cdot 5^{2} \cdot 17^{c} \cdot 13^{d} \cdot 7^{e} \cdot 11^{f} \cdot w^{\prime \prime}, \quad(c \geq 2)
$$

and

$$
2^{6} \cdot 5 \cdot 17^{c-1} \cdot 13^{d-1} \cdot 7^{e} \cdot 11^{f} \cdot w^{\prime \prime}=\sigma^{* *}\left(17^{c}\right) \cdot \sigma^{* *}\left(13^{d}\right) \cdot \sigma^{* *}\left(7^{e}\right) \cdot \sigma^{* *}\left(11^{f}\right) \sigma^{* *}\left(w^{\prime \prime}\right),
$$

where

$$
w^{\prime \prime} \text { has not more than two odd prime factors and }\left(w^{\prime \prime}, 2.3 .5 .7 .11 .13 .17\right)=1 \text {. }
$$

Let $e=1$. Since $\sigma^{* *}(7)=8$, from $(3.18 b)$ it follows that $w^{\prime \prime}=1$. In this case $n=2^{7} \cdot 5^{2} \cdot 17^{c} \cdot 13^{d} \cdot 7^{e} \cdot 11^{f}$. Hence

$$
3=\frac{\sigma^{* *}(n)}{n}<\frac{255}{128} \cdot \frac{26}{25} \cdot \frac{17}{16} \cdot \frac{13}{12} \cdot \frac{8}{7} \cdot \frac{11}{10}=2.998052455<3,
$$

a contradiction. We have

$$
\sigma^{* *}\left(7^{e}\right)= \begin{cases}50 & \text { when } e=2 \\ 400 & \text { when } e=3 \\ 2^{6} .43 & \text { when } e=4\end{cases}
$$

If $e=2$ or $e=3,5^{2} \mid \sigma^{* *}\left(7^{e}\right)$. Hence $5^{2}$ is a factor of the left-hand side of (3.18b). This is not possible. When $e=4,2^{6} \mid \sigma^{* *}\left(7^{e}\right)$. Thus $2^{9}$ is a factor of the right-hand side of $(3.18 b)$, whereas $2^{6}$ is a unitary divisor of its left-hand side.

Thus we may assume that $e \geq 5$.

We now prove that $c=2$ is not admissible in (3.18b). We assume that $c=2$ and obtain a contradiction by examining the prime factors of $\sigma^{* *}\left(7^{e}\right)$. Since $\sigma^{* *}\left(17^{2}\right)=290=2.5 .29$, taking $c=2$ in $(3.18 b)$, we find that $29 \mid w^{\prime \prime}$. Let $w^{\prime \prime}=29^{g} . w^{*}$. Using this in $(3.18 a)(c=2)$ and $(3.18 b)$ $(c=2)$, we get

$$
n=2^{7} \cdot 5^{2} \cdot 17^{2} \cdot 13^{d} \cdot 7^{e} \cdot 11^{f} \cdot 29^{g} \cdot w^{*},
$$

and

$$
2^{5} \cdot 17 \cdot 13^{d-1} \cdot 7^{e} \cdot 11^{f} 29^{g-1} \cdot w^{*}=\sigma^{* *}\left(13^{d}\right) \cdot \sigma^{* *}\left(7^{e}\right) \cdot \sigma^{* *}\left(11^{f}\right) \cdot \sigma^{* *}\left(29^{g}\right) \cdot \sigma^{* *}\left(w^{*}\right),
$$

where

$$
w^{*} \text { has not more than one odd prime factors and }\left(w^{*}, 2.3 .5 .7 .11 .13 .17\right)=1 \text {. }
$$


If $e$ is odd or $4 \mid e$, then $8 \mid \sigma^{* *}\left(7^{e}\right)$; from $(3.19 b)$ we find that this results in an imbalance of powers of 2 between both sides of (3.19b). Hence we may assume that $e=2 k$, where $k$ is odd and $k \geq 3$, since $e \geq 5$. We have

$$
\sigma^{* *}\left(7^{e}\right)=\left(\frac{7^{k}-1}{6}\right) \cdot\left(7^{k+1}+1\right) .
$$

We obtain a contradiction by showing that $\frac{7^{k}-1}{6}$ and $\frac{7^{k+1}+1}{2}$ are divisible by two distinct odd primes $p^{\prime}$ and $q^{\prime}$, respectively, which are also factors of $w^{*}$. This would contradict $(3.19 c)$.

In Lemma 2.4 (a), if we replace the interval $[3,2520]$ by $[3,31]$ we have the following conclusion:

(I) If $p \mid 7^{k}-1$, where $p \in[3,31]-\{3,19\}$ and $\operatorname{ord}_{p} 7$ is odd, then we can find an odd prime $p^{\prime} \mid \frac{7^{k}-1}{6}$ and $p^{\prime}>31$. By $(3.19 b), p^{\prime} \mid w^{*}$.

Let

$$
S_{7}^{\prime}=\left\{p \mid 7^{k}-1: p \in[3,31]-\{3,19\} \text { and } \operatorname{ord}_{p} 7 \text { is odd }\right\} .
$$

If $S_{7}^{\prime}$ is non-empty, we can conclude from (I) that $w^{*}$ is divisible by an odd prime $p^{\prime} \mid \frac{7^{k}-1}{6}$. Suppose that $S_{7}^{\prime}$ is empty. Since $p \nmid 7^{k}-1$ if $\operatorname{ord}_{p} 7$ is even, it follows that $7^{k}-1$ is not divisible by any prime in $[3,31]$ except for possibly 3 and 19 .

We have $19\left|7^{k}-1 \Longleftrightarrow 3\right| k \Longleftrightarrow 9 \mid 7^{k}-1$. Also, $9\left|7^{k}-1 \Longleftrightarrow 3\right| \frac{7^{k}-1}{6}$. Further from $(3.19 b), 3 \mid \frac{7^{k}-1}{6} \sigma^{* *}\left(7^{e}\right)$ implies that 3 is a factor of the left-hand side of $(3.19 b)$ and so $3 \mid w^{*}$. This cannot happen. Hence $9 \nmid 7^{k}-1$ and consequently $19 \nmid 7^{k}-1$.

Thus $\frac{7^{k}-1}{6}$ is not divisible by 3 and 19 . It follows that $\frac{7^{k}-1}{6}$ is not divisible by any prime in $[3,31]$; also, $\frac{7^{k}-1}{6}>1$ and odd. Let $p^{\prime} \mid \frac{7^{k}-1}{6}$. Then $p^{\prime}>31$ and from $(3.19 b), p^{\prime} \mid w^{*}$.

In Lemma 2.4 (b), if we replace the interval $[3,1193]$ by $[3,31]$ we have the following conclusion:

(II) If $q \mid 7^{k+1}+1, q \in[3,31]-\{5,13\}$ and $s=\frac{1}{2} \operatorname{ord}_{p} 7$ is even, then we can find an odd prime $q^{\prime} \mid \frac{7^{k+1}+1}{2}$ and $q^{\prime}>31$. By $(3.19 b), q^{\prime} \mid w^{*}$.

Let

$$
T_{7}^{\prime}=\left\{q \mid 7^{k+1}+1: q \in[3,31]-\{5,13\} \text { and } s=\frac{1}{2} \operatorname{ord}_{p} 7 \text { is even }\right\} .
$$

If $T_{7}^{\prime}$ is non-empty, by (II) above, we can find an odd prime $q^{\prime} \mid \frac{7^{k+1}+1}{2}$ and $q^{\prime}>31$. Also, $q^{\prime} \mid w^{*}$. This is what we require.

Suppose that $T_{7}^{\prime}$ is empty. Since $q \nmid 7^{k+1}+1$ if $s=\frac{1}{2} \operatorname{ord}_{p} 7$ is not even, it follows that $\frac{7^{k+1}+1}{2}$ is not divisible by any prime in $[3,31]$ except for possibly 5 and 13 .

If $5\left|\frac{7^{k+1}+1}{2}\right| \sigma^{* *}\left(7^{e}\right)$, then from $(3.19 b)$ it follows that 5 divides its left-hand side. This is not possible. Hence $5 \nmid 7^{k+1}+1$. 
Suppose $13 \mid 7^{k+1}+1$. This is equivalent to $k+1=6 u$. Hence $7^{6}+1 \mid 7^{k+1}+1$. Also, $7^{6}+1=2.5^{2} \cdot 13.181$. Hence $5\left|7^{6}+1\right| 7^{k+1}+1$. But this is false.

Thus $7^{k+1}+1$ is not divisible by 5 and 13 and so $\frac{7^{k+1}+1}{2}$ is not divisible by any prime in $[3,31]$. Since $\frac{7^{k+1}+1}{2}>1$ and odd, let $q^{\prime} \mid \frac{7^{k+1}+1}{2}$. Then $q^{\prime}>31$ and from $(3.19 b), q^{\prime} \mid w^{*}$.

It follows that $w^{*}$ is divisible by two distinct odd primes $p^{\prime}$ and $q^{\prime}$. But this is not possible by $(3.19 c)$.

This proves that $c=2$ is not admissible. We may assume that $c \geq 3$. The relevant equations are $(3.18 a)$ and $(3.18 b)$ with $c \geq 3$ and $e \geq 5$. By Lemma 2.1, we have $\frac{\sigma^{* *}\left(17^{c}\right)}{17^{c}} \geq \frac{88452}{83521}$ $(c \geq 3), \frac{\sigma^{* *}\left(13^{d}\right)}{13^{d}} \geq \frac{30772}{28561}(d \geq 3), \frac{\sigma^{* *}\left(7^{e}\right)}{7^{e}} \geq \frac{136914}{117649}(e \geq 5)$ and $\frac{\sigma^{* *}\left(11^{f}\right)}{11^{f}} \geq \frac{15984}{14641}(f \geq 3)$.

If $d \geq 3$ and $f \geq 3$, from $(3.18 a)$, we have

$$
3=\frac{\sigma^{* *}(n)}{n} \geq \frac{255}{128} \cdot \frac{26}{25} \cdot \frac{88452}{83521} \cdot \frac{30772}{28561} \cdot \frac{136914}{117649} \cdot \frac{15984}{14641}=3.00353146>3,
$$

a contradiction. Hence $d \geq 3$ and $f \geq 3$ cannot hold together. So we have the following cases:

- Case 1: $\{d \geq 3, f=1,2\}$

- Case 2: $\{d=1,2, f \geq 3\}$, and

- Case 3: $\{d=1,2, f=1,2\}$.

Let $f=1$. Taking $f=1$ in $(3.18 b)$, since $\sigma^{* *}(11)=12$, it follows that 3 is a factor of the left-hand side of $(3.18 b)$. Since $3 \nmid n$, by our assumption, this is not possible.

Let $f=2$. We have $\sigma^{* *}\left(11^{2}\right)=122=2.61$. From $(3.18 b)(f=2), 61 \mid w^{\prime \prime}$. Let $w^{\prime \prime}=61^{g} . w^{*}$. From $(3.18 a)(f=2)$ and $(3.18 b)(f=2)$, we obtain

$$
n=2^{7} \cdot 5^{2} \cdot 17^{c} \cdot 13^{d} \cdot 7^{e} \cdot 11^{2} \cdot 61^{g} \cdot w^{*}, \quad(c \geq 3)
$$

and

$$
2^{5} \cdot 5 \cdot 17^{c-1} \cdot 13^{d-1} \cdot 7^{e} \cdot 11^{2} \cdot 61^{g-1} \cdot w^{*}=\sigma^{* *}\left(17^{c}\right) \cdot \sigma^{* *}\left(13^{d}\right) \cdot \sigma^{* *}\left(7^{e}\right) \cdot \sigma^{* *}\left(61^{g}\right) \cdot \sigma^{* *}\left(w^{*}\right),
$$

where

$$
w^{*}=1 \text { or a prime power with }\left(w^{*}, 2.3 .5 .7 .11 .13 .17\right)=1 .
$$

We now show that $19 \nmid w^{*}$. On the contrary, suppose that $19 \mid w^{*}$ so that $w^{*}=19^{h}$. Using this in $(3.20 a)$ and $(3.20 b)$, we get

$$
n=2^{7} \cdot 5^{2} \cdot 17^{c} \cdot 13^{d} \cdot 7^{e} \cdot 11^{2} \cdot 61^{g} \cdot w^{*}, \quad(c \geq 3)
$$

and

$$
2^{5} \cdot 5 \cdot 17^{c-1} \cdot 13^{d-1} \cdot 7^{e} \cdot 11^{2} \cdot 61^{g} \cdot 19^{h}=\sigma^{* *}\left(17^{c}\right) \cdot \sigma^{* *}\left(13^{d}\right) \cdot \sigma^{* *}\left(7^{e}\right) \cdot \sigma^{* *}\left(61^{g}\right) \cdot \sigma^{* *}\left(19^{h}\right) .
$$

If $e$ is odd or $4 \mid e$, then $8 \mid \sigma^{* *}\left(7^{e}\right)$; this brings an imbalance in powers of 2 between the two sides of $(3.21 b)$. We may thus assume that $e=2 k$, where $k$ is odd and $k \geq 3$ (since $e \geq 5$ ). So, $\sigma^{* *}\left(7^{e}\right)=\left(\frac{7^{k}-1}{6}\right) \cdot\left(7^{k+1}+1\right)$. 
Since $k$ is odd and $k \geq 3, \frac{7^{k}-1}{6}>1$ and odd. Also, $7^{k}-1$ is not divisible by $5,11,13,17$ and 61 , since $k$ is odd.

Further, $9\left|7^{k}-1 \Longleftrightarrow 3\right| k \Longleftrightarrow 19 \mid 7^{k}-1$. But $9 \mid 7^{k}-1$ implies that $3\left|\frac{7^{k}-1}{6}\right| \sigma^{* *}\left(7^{e}\right)$ and from $(3.21 b)$, we find that this is not possible. Hence $9 \nmid 7^{k}-1$ and consequently $19 \nmid 7^{k}-1$.

Thus $\frac{7^{k}-1}{6}>1$, is odd and not divisible by $5,7,11,13,17,19$ and 61 (divisible by none of these primes). From (3.21b), this is not possible. This contradiction shows that $19 \nmid w^{*}$.

From $(3.20 c)$, we may assume that $w^{*}=p^{h}$, where $p \geq 23$. From $(3.20 a)$, we have $n=$ $2^{7} \cdot 5^{2} \cdot 17^{c} \cdot 13^{d} \cdot 7^{e} \cdot 11^{2} \cdot 61^{g} \cdot p^{h}$. Hence

$$
3=\frac{\sigma^{* *}(n)}{n}<\frac{255}{128} \cdot \frac{26}{25} \cdot \frac{17}{16} \cdot \frac{13}{12} \cdot \frac{7}{6} \cdot \frac{122}{121} \cdot \frac{61}{60} \cdot \frac{23}{22}=2.981670063<3,
$$

a contradiction.

Hence $f=2$ is not admissible.

Let $d=1$ and $f \geq 3$. Already $c \geq 3$ and $e \geq 5$. From $(3.18 a)(d=1)$, we have

$$
3=\frac{\sigma^{* *}(n)}{n} \geq \frac{255}{128} \cdot \frac{26}{25} \cdot \frac{88452}{83521} \cdot \frac{14}{13} \cdot \frac{15984}{14641} \cdot \frac{136914}{117649}=3.002164976>3,
$$

a contradiction.

Let $d=2$. Since $\sigma^{* *}\left(13^{2}\right)=170=2.5 .17$. Taking $d=2$ in $(3.18 a)$ and $(3.18 b)$, we obtain

$$
n=2^{7} \cdot 5^{2} \cdot 17^{c} \cdot 13^{2} \cdot 7^{e} \cdot 11^{f} \cdot w^{\prime \prime},
$$

and

$$
2^{5} \cdot 17^{c-2} \cdot 13 \cdot 7^{e} \cdot 11^{f} \cdot w^{\prime \prime}=\sigma^{* *}\left(17^{c}\right) \cdot \sigma^{* *}\left(7^{e}\right) \cdot \sigma^{* *}\left(11^{f}\right) \cdot \sigma^{* *}\left(w^{\prime \prime}\right),
$$

where

$w^{\prime \prime}$ has not more than two odd prime factors and $\left(w^{\prime \prime}, 2.3 .5 .7 .11 .13 .17\right)=1$.

By applying Lemma 2.4, we show that $\sigma^{* *}\left(7^{e}\right)$ is divisible by two distinct odd primes each greater than 67 when $e$ is even and $4 \nmid e$.

If $e$ is odd or $4 \mid e$, then $8 \mid \sigma^{* *}\left(7^{e}\right)$. From $(3.22 b)$, it follows that $w^{\prime \prime}=1$. Hence from $(3.22 a)$, $n=2^{7} \cdot 5^{2} \cdot 17^{c} .13^{2} .7^{e} .11^{f}$ and so

$$
3=\frac{\sigma^{* *}(n)}{n}<\frac{255}{128} \cdot \frac{26}{25} \cdot \frac{17}{16} \cdot \frac{170}{169} \cdot \frac{7}{6} \cdot \frac{11}{10}=2.841804387<3
$$

a contradiction.

We may assume that $e=2 k$, where $k$ is odd and $\geq 3$. We have

$$
\sigma^{* *}\left(7^{e}\right)=\left(\frac{7^{k}-1}{6}\right) \cdot\left(7^{k+1}+1\right)
$$

We show (by using Lemma 2.4) that:

(III) $\frac{7^{k}-1}{6}$ is divisible by an odd prime $p^{\prime} \mid w^{\prime \prime}$ and $p^{\prime}>67$,

(IV) $\frac{7^{k+1}+1}{2}$ is divisible by an odd prime $q^{\prime} \mid w^{\prime \prime}$ and $q^{\prime}>67$. 
(III) Proof of (III). If we replace the interval $[3,2520]$ by the interval $[3,67]$ in Lemma 2.4 (a), then we have the following conclusion:

(A) If $p \in[3,67]-\{3,19,37\}, p \mid 7^{k}-1$ and $\operatorname{ord}_{7} p$ is odd, then we can find an odd prime $p^{\prime} \mid \frac{7^{k}-1}{6}$ and $p^{\prime}>67$.

Let

$$
S_{7}^{\prime}=\left\{p \mid 7^{k}-1: p \in[3,67]-\{3,19,37\} \text { and } \operatorname{ord}_{p} 7 \text { is odd }\right\} .
$$

By (A), if $S_{7}^{\prime}$ is non-empty, then (III) holds.

Suppose that $S_{7}^{\prime}$ is empty. Since $p \nmid 7^{k}-1$ if $\operatorname{ord}_{7} p$ is even, it follows that $\frac{7^{k}-1}{6}$ is not divisible by any prime in $[3,67]$ except for possibly 3,19 and 37 . We have the following:

(i) $9\left|7^{k}-1 \Longleftrightarrow 3\right| \frac{7^{k}-1}{6}$. Hence if $9 \mid 7^{k}-1$, then 3 is a factor of the left-hand side of $(3.22 b)$ which is not the case. Hence $9 \nmid 7^{k}-1$ and so $\frac{7^{k}-1}{6}$ is not divisible by 3 .

(ii) $19\left|7^{k}-1 \Longleftrightarrow 3\right| k \Longleftrightarrow 9 \mid 7^{k}-1$. By (i), $19 \nmid 7^{k}-1$.

(iii) Suppose $37 \mid 7^{k}-1$. Hence $9 \mid k$ and so $7^{9}-1 \mid 7^{k}-1$. Also, $7^{9}-1=2.3^{3} .19 .37 .1063$. Hence $19\left|7^{9}-1\right| 7^{k}-1$. By (i) this is false. Hence $37 \nmid 7^{k}-1$.

(iv) Since $k$ is odd and $\geq 3, \frac{7^{k}-1}{6}$ is odd and $>1$.

From the above discussion, it is clear that $\frac{7^{k}-1}{6}>1$, is odd and is not divisible by any prime in $[3,67]$. Let $p^{\prime} \mid \frac{7^{k}-1}{6}$. Then $p^{\prime}>67$ and by $(3.22 b), p^{\prime} \mid w^{\prime \prime}$. This proves (III).

(IV) Proof of (IV). If we replace the interval $[3,1193]$ by the interval $[3,67]$ in Lemma 2.4 (b), then we have the following conclusion:

(B) If $q \in[3,67]-\{5,13\}, q \mid 7^{k+1}+1$ and $s=\frac{1}{2} \operatorname{ord}_{7} q$ is even, then we can find an odd prime $q^{\prime} \mid \frac{7^{k+1}+1}{2}$ and $q^{\prime}>67$.

Let

$$
T_{7}^{\prime}=\left\{q \mid 7^{k+1}+1: q \in[3,67]-\{5,13\} \text { and } s=\frac{1}{2} \operatorname{ord}_{q} 7 \text { is even }\right\} .
$$

By (B), if $T_{7}^{\prime}$ is non-empty, then (III) holds.

Suppose that $T_{7}^{\prime}$ is empty. Since $q \nmid 7^{k+1}+1$ if $s=\frac{1}{2} \operatorname{ord}_{q} 7$ is not even, it follows that $\frac{7^{k+1}+1}{2}$ is not divisible by any prime in $[3,67]$ except for possibly by 5 and 13 .

From $(3.22 b)$ it follows that $5 \nmid \sigma^{* *}\left(7^{e}\right)$, since 5 is not a factor of the left-hand side of $(3.22 b)$.

Note that $5 \mid 7^{k+1}+1$ implies that $5^{2} \mid \sigma^{* *}\left(7^{e}\right)$; also, $13 \mid 7^{k+1}+1$ implies that $k+1=6 u$ and so $5^{2}\left|7^{6}+1\right| 7^{k+1}+1 \mid \sigma^{* *}\left(7^{e}\right)$. In both the cases $5^{2} \mid \sigma^{* *}\left(7^{e}\right)$ which is false. Thus $7^{k+1}+1$ is divisible by neither 5 nor 13 .

It follows that $\frac{7^{k+1}+1}{2}$ is not divisible by any prime in [3,67]. Also, $\frac{7^{k+1}+1}{2}>1$ and is odd. Let $q^{\prime} \mid \frac{7^{k+1}+1}{2}$. Then $q^{\prime}>67$ and $q^{\prime} \mid w^{\prime \prime}$ by $(3.22 b)$.

This proves (IV). 
From $(3.22 c)$, we have $w^{\prime \prime}=\left(p^{\prime}\right)^{g} \cdot\left(q^{\prime}\right)^{h}$. Hence from $(3.22 a)$, we have

$$
n=2^{7} \cdot 5^{2} \cdot 17^{c} \cdot 13^{2} \cdot 7^{e} \cdot 11^{f} \cdot\left(p^{\prime}\right)^{g} \cdot\left(q^{\prime}\right)^{h}
$$

so that

a contradiction.

$$
3=\frac{\sigma^{* *}(n)}{n}<\frac{255}{128} \cdot \frac{26}{25} \cdot \frac{17}{16} \cdot \frac{170}{169} \cdot \frac{7}{6} \cdot \frac{11}{10} \cdot \frac{71}{70} \cdot \frac{73}{72}=2.922434948<3,
$$

Hence $d=2$ is not possible. Thus we proved the non-admissibility of $f=1,2$ and $d=1,2$. These cover the three cases (Case 1, Case 2 and Case 3) mentioned below (3.19d).

This completes the proof of $11 \nmid n$.

Let $s=19$ or 23 . We show that $s \nmid n$ which is same as $s \nmid w^{\prime}$ where $n$ is as in $(3.17 a)$. On the contrary, we assume that $s \mid w^{\prime}$ so that $w^{\prime}=s^{f} . w^{\prime \prime}$. From $(3.17 a)$ and $(3.17 b)$, we have

$$
n=2^{7} \cdot 5^{2} \cdot 17^{c} \cdot 13^{d} \cdot 7^{e} \cdot s^{f} \cdot w^{\prime \prime}, \quad(c \geq 2)
$$

and

$$
2^{6} \cdot 5 \cdot 17^{c-1} \cdot 13^{d-1} \cdot 7^{e} \cdot s^{f} \cdot w^{\prime \prime}=\sigma^{* *}\left(17^{c}\right) \cdot \sigma^{* *}\left(13^{d}\right) \cdot \sigma^{* *}\left(7^{e}\right) \cdot \sigma^{* *}\left(s^{f}\right) \cdot \sigma^{* *}\left(w^{\prime \prime}\right),
$$

where

$$
w^{\prime \prime} \text { has not more than two odd prime factors and }\left(w^{\prime}, 2.3 .5 .7 .11 .13 .17 . s\right)=1 \text {. }
$$

We obtain a contradiction by examining $\sigma^{* *}\left(7^{e}\right)$. If $e$ is odd or $4 \mid e$, then $8 \mid \sigma^{* *}\left(7^{e}\right)$. From $(3.23 b)$, we infer that $w^{\prime \prime}=1$. From $(3.23 a), n=2^{7} \cdot 5^{2} \cdot 17^{c} \cdot 13^{d} \cdot 7^{e} \cdot s^{f}$ where $s \geq 19$; and so

$$
3=\frac{\sigma^{* *}(n)}{n}<\frac{255}{128} \cdot \frac{26}{25} \cdot \frac{17}{16} \cdot \frac{13}{12} \cdot \frac{7}{6} \cdot \frac{19}{18}=2.936854836<3,
$$

a contradiction.

We may assume that $e=2 k$, where $k$ is odd, and since $e \neq 2, k \geq 3$. Also,

$$
\sigma^{* *}\left(7^{e}\right)=\left(\frac{7^{k}-1}{6}\right) \cdot\left(7^{k+1}+1\right) \quad(k \text { odd, } k \geq 3) .
$$

We show that:

(III) $)^{\prime} \frac{7^{k}-1}{6}$ is divisible by a prime $p^{\prime}>89$ and $p^{\prime} \mid w^{\prime \prime}$,

$(\mathrm{IV})^{\prime} \frac{7^{k+1}+1}{2}$ is divisible by a prime $q^{\prime}>89$ and $q^{\prime} \mid w^{\prime \prime}$.

The left-hand side of $(3.23 b))$ is neither divisible by 3 nor $5^{2}$. The proofs of (III) ${ }^{\prime}$ and (IV) $)^{\prime}$ are similar to those of (III) and (IV); we need to apply Lemma 2.4 by replacing the intervals $[3,2520]$ and $[3,1193]$ by $[3,89]$. We omit the details.

From $(3.23 c)$, we have $w^{\prime \prime}=\left(p^{\prime}\right)^{g} \cdot\left(q^{\prime}\right)^{h}$. We may assume that $p^{\prime} \geq 97$ and $q^{\prime} \geq 101$. From (3.23a), we have $n=2^{7} \cdot 5^{2} \cdot 17^{c} \cdot 13^{d} \cdot 7^{e} \cdot s^{f} \cdot\left(p^{\prime}\right)^{g} \cdot\left(q^{\prime}\right)^{h}$, so that

$$
3=\frac{\sigma^{* *}(n)}{n}<\frac{255}{128} \cdot \frac{26}{25} \cdot \frac{17}{16} \cdot \frac{13}{12} \cdot \frac{7}{6} \cdot \frac{19}{18} \cdot \frac{97}{96} \cdot \frac{101}{100}=2.997121544<3,
$$

a contradiction. Hence $s \nmid w^{\prime \prime}$.

Thus if $n$ is as in (3.3a), $3 \nmid n$ and $7 \mid n$, then $n$ is divisible by none of 11,19 and 23 . The proof of (b) of Lemma 3.9 is complete. 
Lemma 3.10. Let $n$ be as in (3.3a) with $c \geq 2$. Assume that $3 \nmid n$. If $7 \mid n$, then $n$ cannot be a bi-unitary triperfect number.

Proof. Suppose that $7 \mid n$ and $n$ is a bi-unitary triperfect number. Then $n$ satisfies the equations $(3.17 a)-(3.17 c)$. We obtain a contradiction by examining the factors of $\sigma^{* *}\left(7^{e}\right)$ in $(3.17 b)$.

If $e$ is odd or $4 \mid e$, then $8 \mid \sigma^{* *}\left(7^{e}\right)$. From $(3.17 b)$, we at once have $w^{\prime}=p^{f}$, where $p \geq 29$ by Lemma 3.9 (b). Hence from $(3.17 a), n=2^{7} \cdot 5^{2} \cdot 17^{c} \cdot 13^{d} \cdot 7^{e} \cdot p^{f}$ and so by Lemma 2.1,

$$
3=\frac{\sigma^{* *}(n)}{n}<\frac{255}{128} \cdot \frac{26}{25} \cdot \frac{17}{16} \cdot \frac{13}{12} \cdot \frac{7}{6} \cdot \frac{29}{28}=2.881650798<3
$$

a contradiction.

Let $e=2 k$, where $k$ is odd and $k \geq 3$ (as $e \neq 2$ ). We note that the left-hand side of $(3.17 b)$ is neither divisible by 3 nor $5^{2}$. As in (III) ${ }^{\prime}$ and (IV) $)^{\prime}$ of Lemma 3.9 (b), $\frac{7^{k}-1}{6}$ and $\frac{7^{k+1}+1}{2}$ are divisible by odd primes $p^{\prime}>89$ and $q^{\prime}>89$, respectively.

Further, $w^{\prime}$ is divisible by $p^{\prime}$ and $q^{\prime}$. We may assume that $p^{\prime} \geq 97$ and $q^{\prime} \geq 101$. Assuming that $y$ is a possible third prime factor of $w^{\prime}$ by $(3.17 c)$, by Lemma 3.9 (b), we have $y \geq 29$ and $w^{\prime}=\left(p^{\prime}\right)^{f} \cdot\left(q^{\prime}\right)^{g} \cdot y^{h}$. By $(3.17 c)$ and $(3.17 a), n=2^{7} \cdot 5^{2} \cdot 17^{c} \cdot 13^{d} \cdot 7^{e} \cdot\left(p^{\prime}\right)^{f} \cdot\left(q^{\prime}\right)^{g} \cdot y^{h}$ and by Lemma 2.1, we have

$$
3=\frac{\sigma^{* *}(n)}{n}<\frac{255}{128} \cdot \frac{26}{25} \cdot \frac{17}{16} \cdot \frac{13}{12} \cdot \frac{7}{6} \cdot \frac{97}{96} \cdot \frac{101}{100} \cdot \frac{29}{28}=2.940784673<3,
$$

a contradiction. Hence $n$ cannot be a bi-unitary triperfect number. The proof of Lemma 3.10 is complete.

Completion of Proof of Theorem 3.1(b). Theorem 3.1(b) follows from Lemmas 3.1-3.10.

Remark 3.4. Let $n$ be as given in (3.1a) and $b \geq 3$. Assume that $n$ is a bi-unitary triperfect number. Then $(3.1 b)$ is valid. Further suppose that $n$ is not divisible by 3 . If $b$ is odd or $4 \mid b$, then $3 \mid \sigma^{* *}\left(5^{b}\right)$. Also, if $c$ is odd or $4 \mid c$, then $9 \mid \sigma^{* *}\left(17^{c}\right)$. These are not possible in $(3.1 b)$, and therefore it follows that $b=2 k$ and $c=2 \ell$, where $k \geq 3$ and $\ell$ are odd. Hence $b \geq 6$ and $c \geq 2$. We will consider the case $3 \nmid n$ in more detail in future.

\section{References}

[1] Hagis, P., Jr. (1987). Bi-unitary amicable and multiperfect numbers, The Fibonacci Quarterly, 25(2), 144-150.

[2] Haukkanen, P., \& Sitaramaiah, V. (2020). Bi-unitary multiperfect numbers, I, Notes on Number Theory and Discrete Mathematics, 26(1), 93-171.

[3] Haukkanen, P., \& Sitaramaiah, V. (2020). Bi-unitary multiperfect numbers, II, Notes on Number Theory and Discrete Mathematics, 26(2), 1-26. 
[4] Haukkanen, P., \& Sitaramaiah, V. (2020). Bi-unitary multiperfect numbers, III, Notes on Number Theory and Discrete Mathematics, 26(3), 33-67.

[5] Sándor, J., \& Crstici, P. (2004). Handbook of Number Theory II, Kluwer Academic.

[6] Suryanarayana, D. (1972). The number of bi-unitary divisors of an integer, In: The Theory of Arithmetic Functions, Lecture Notes in Mathematics, 251, 273-282, New York, SpringerVerlag.

[7] Wall, C. R. (1972). Bi-unitary perfect numbers, Proceedings of the American Mathematical Society, 33(1), 39-42. 


\section{Appendix A Tables of $\operatorname{ord}_{p} 13$}

Let $p$ denote an odd prime $\neq 13$. In the following table, $r$ denotes the smallest positive integer such that $13^{r} \equiv 1(\bmod p)$; that is, $r=\operatorname{ord}_{p} 13 ; s$ denotes the smallest positive integer such that $13^{s} \equiv-1(\bmod p)$ if $s$ exists; if $s$ does not exist, that is, if $13^{t}+1$ is not divisible by $p$ for any positive integer $t$, the entry in column $s$ will be filled up by dash sign. If $r$ is even, then $s=r / 2$, and if $r$ is odd $s$ does not exist.

\begin{tabular}{|c|c|c|c|c|c|c|c|c|c|c|c|}
\hline SL.No & $p$ & $r$ & $s$ & SL.No & $p$ & $r$ & $s$ & SL.No & $p$ & $r$ & $s$ \\
\hline 1 & 3 & 1 & - & 37 & 163 & 54 & 27 & 73 & 373 & 62 & 31 \\
2 & 5 & 4 & 2 & 38 & 167 & 166 & 83 & 74 & 379 & 378 & 189 \\
3 & 7 & 2 & 1 & 39 & 173 & 86 & 43 & 75 & 383 & 382 & 191 \\
4 & 11 & 10 & 5 & 40 & 179 & 89 & - & 76 & 389 & 97 & - \\
5 & 13 & - & - & 41 & 181 & 45 & - & 77 & 397 & 396 & 198 \\
6 & 17 & 4 & 2 & 42 & 191 & 95 & - & 78 & 401 & 400 & 200 \\
7 & 19 & 18 & 9 & 43 & 193 & 64 & 32 & 79 & 409 & 136 & 68 \\
8 & 23 & 11 & - & 44 & 197 & 196 & 98 & 80 & 419 & 11 & - \\
9 & 29 & 14 & 7 & 45 & 199 & 99 & - & 81 & 421 & 20 & 10 \\
10 & 31 & 30 & 15 & 46 & 211 & 35 & - & 82 & 431 & 430 & 215 \\
11 & 37 & 36 & 18 & 47 & 223 & 74 & 37 & 83 & 433 & 216 & 108 \\
12 & 41 & 40 & 20 & 48 & 227 & 226 & 113 & 84 & 439 & 219 & - \\
13 & 43 & 21 & - & 49 & 229 & 76 & 38 & 85 & 443 & 17 & - \\
14 & 47 & 46 & 23 & 50 & 233 & 116 & 58 & 86 & 449 & 448 & 224 \\
15 & 53 & 13 & - & 51 & 239 & 238 & 119 & 87 & 457 & 456 & 228 \\
16 & 59 & 58 & 29 & 52 & 241 & 240 & 120 & 88 & 461 & 92 & 46 \\
17 & 61 & 3 & - & 53 & 251 & 125 & - & 89 & 463 & 42 & 21 \\
18 & 67 & 66 & 33 & 54 & 257 & 128 & 64 & 90 & 467 & 233 & - \\
19 & 71 & 70 & 35 & 55 & 263 & 131 & - & 91 & 479 & 478 & 239 \\
20 & 73 & 72 & 36 & 56 & 269 & 134 & 67 & 92 & 487 & 486 & 243 \\
21 & 79 & 39 & - & 57 & 271 & 18 & 9 & 93 & 491 & 245 & - \\
22 & 83 & 82 & 41 & 58 & 277 & 46 & 23 & 94 & 499 & 166 & 83 \\
23 & 89 & 88 & 44 & 59 & 281 & 280 & 140 & 95 & 503 & 251 & - \\
24 & 97 & 96 & 48 & 60 & 283 & 141 & - & 96 & 509 & 508 & 254 \\
25 & 101 & 50 & 25 & 61 & 293 & 292 & 146 & 97 & 521 & 260 & 130 \\
26 & 103 & 17 & - & 62 & 307 & 306 & 153 & 98 & 523 & 261 & - \\
27 & 107 & 53 & - & 63 & 311 & 31 & - & 99 & 541 & 540 & 270 \\
28 & 109 & 108 & 54 & 64 & 313 & 156 & 78 & 100 & 547 & 21 & - \\
29 & 113 & 56 & 28 & 65 & 317 & 316 & 158 & 101 & 557 & 556 & 278 \\
30 & 127 & 63 & - & 66 & 331 & 66 & 33 & 102 & 563 & 281 & - \\
31 & 131 & 65 & - & 67 & 337 & 21 & - & 103 & 569 & 284 & 142 \\
32 & 137 & 136 & 68 & 68 & 347 & 173 & - & 104 & 571 & 285 & - \\
33 & 139 & 69 & - & 69 & 349 & 348 & 174 & 105 & 577 & 576 & 288 \\
34 & 149 & 148 & 74 & 70 & 353 & 352 & 176 & 106 & 587 & 586 & 293 \\
35 & 151 & 150 & 75 & 71 & 359 & 358 & 179 & 107 & 593 & 592 & 296 \\
36 & 157 & 6 & 3 & 72 & 367 & 183 & - & 108 & 599 & 299 & - \\
\hline
\end{tabular}




\section{Appendix B Tables of ord $_{p} 17$}

Let $p$ denote an odd prime $\neq 17$. In the following table, $r$ denotes the smallest positive integer such that $17^{r} \equiv 1(\bmod p)$; that is, $r=\operatorname{ord}_{p} 17 ; s$ denotes the smallest positive integer such that $17^{s} \equiv-1(\bmod p)$ if $s$ exists; if $s$ does not exist, that is, if $17^{t}+1$ is not divisible by $p$ for any positive integer $t$, the entry in column $s$ will be filled up by dash sign. If $r$ is even, then $s=r / 2$, and if $r$ is odd $s$ does not exist.

\begin{tabular}{|c|c|c|c|c|c|c|c|c||c|c|c|c|}
\hline SL.No & $p$ & $r$ & $s$ & SL.No & $p$ & $r$ & $s$ & $S L . N o$ & $p$ & $r$ & $s$ \\
\hline 1 & 3 & 2 & 1 & 33 & 139 & 138 & 69 & 65 & 317 & 316 & 158 \\
2 & 5 & 4 & 2 & 34 & 149 & 37 & - & 66 & 331 & 165 & - \\
3 & 7 & 6 & 3 & 35 & 151 & 75 & - & 67 & 337 & 112 & 56 \\
4 & 11 & 10 & 5 & 36 & 157 & 39 & - & 68 & 347 & 346 & 173 \\
5 & 13 & 6 & 3 & 37 & 163 & 54 & 27 & 69 & 349 & 58 & 29 \\
6 & 17 & - & - & 38 & 167 & 166 & 83 & 70 & 353 & 88 & 44 \\
7 & 19 & 9 & - & 39 & 173 & 172 & 86 & 71 & 359 & 179 & - \\
8 & 23 & 22 & 11 & 40 & 179 & 89 & - & 72 & 367 & 366 & 183 \\
9 & 29 & 4 & 2 & 41 & 181 & 36 & 18 & 73 & 373 & 62 & 31 \\
10 & 31 & 30 & 15 & 42 & 191 & 95 & - & 74 & 379 & 378 & 189 \\
11 & 37 & 36 & 18 & 43 & 193 & 192 & 96 & 75 & 383 & 191 & - \\
12 & 41 & 40 & 20 & 44 & 197 & 196 & 98 & 76 & 389 & 97 & - \\
13 & 43 & 21 & - & 45 & 199 & 66 & - & 77 & 397 & 132 & 66 \\
14 & 47 & 23 & - & 46 & 211 & 210 & 105 & 78 & 401 & 400 & 200 \\
15 & 53 & 26 & 13 & 47 & 223 & 37 & - & 79 & 409 & 51 & - \\
16 & 59 & 29 & - & 48 & 227 & 226 & 113 & 80 & 419 & 418 & 209 \\
17 & 61 & 60 & 30 & 49 & 229 & 19 & - & 81 & 421 & 210 & 105 \\
18 & 67 & 33 & - & 50 & 233 & 232 & 116 & 82 & 431 & 430 & 215 \\
19 & 71 & 10 & 5 & 51 & 239 & 119 & - & 83 & 433 & 27 & - \\
20 & 73 & 24 & 12 & 52 & 241 & 80 & 40 & 84 & 439 & 438 & 219 \\
21 & 79 & 26 & 13 & 53 & 251 & 125 & - & 85 & 443 & 221 & - \\
22 & 83 & 41 & -1 & 54 & 257 & 32 & 16 & 86 & 449 & 448 & 224 \\
23 & 89 & 44 & 22 & 55 & 263 & 131 & - & 87 & 457 & 38 & 19 \\
24 & 97 & 96 & 48 & 56 & 269 & 268 & 134 & 88 & 461 & 230 & 115 \\
25 & 101 & 10 & 5 & 57 & 271 & 135 & - & 89 & 463 & 231 & - \\
26 & 103 & 51 & - & 58 & 277 & 276 & 138 & 90 & 467 & 233 & - \\
27 & 107 & 106 & 53 & 59 & 281 & 140 & 70 & 91 & 479 & 478 & 239 \\
28 & 109 & 36 & 18 & 60 & 283 & 282 & 141 & 92 & 487 & 486 & 243 \\
29 & 113 & 112 & 56 & 61 & 293 & 73 & - & 93 & 491 & 49 & - \\
30 & 127 & 63 & - & 62 & 307 & 3 & - & 94 & 499 & 498 & 249 \\
31 & 131 & 130 & 65 & 63 & 311 & 310 & 155 & 95 & 503 & 502 & 251 \\
32 & 137 & 68 & 34 & 64 & 313 & 312 & 156 & 96 & 509 & 127 & - \\
\hline
\end{tabular}




\section{Appendix C Factors of $13^{t}-1$}

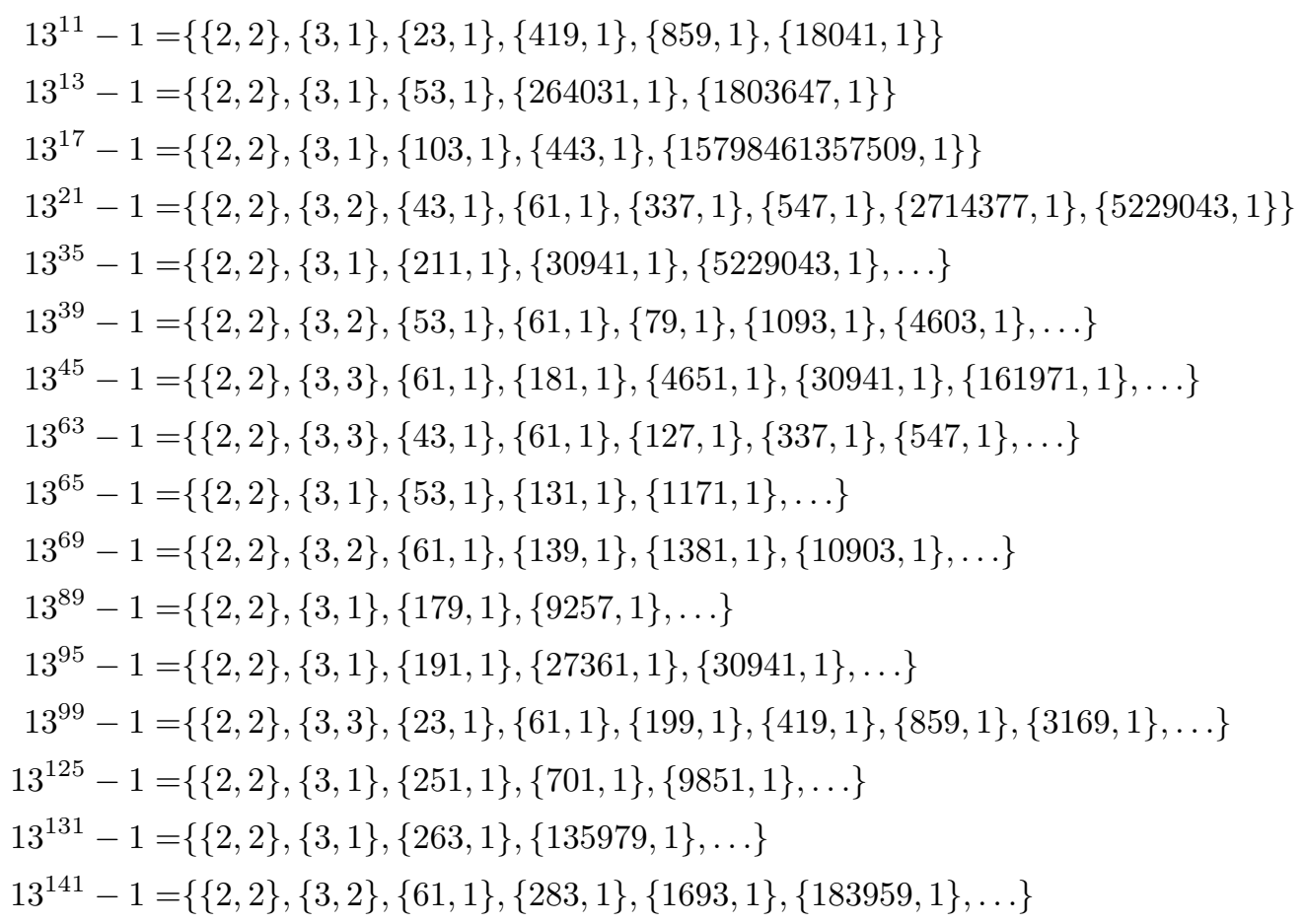

\section{Appendix D Factors of $13^{t}+1$}

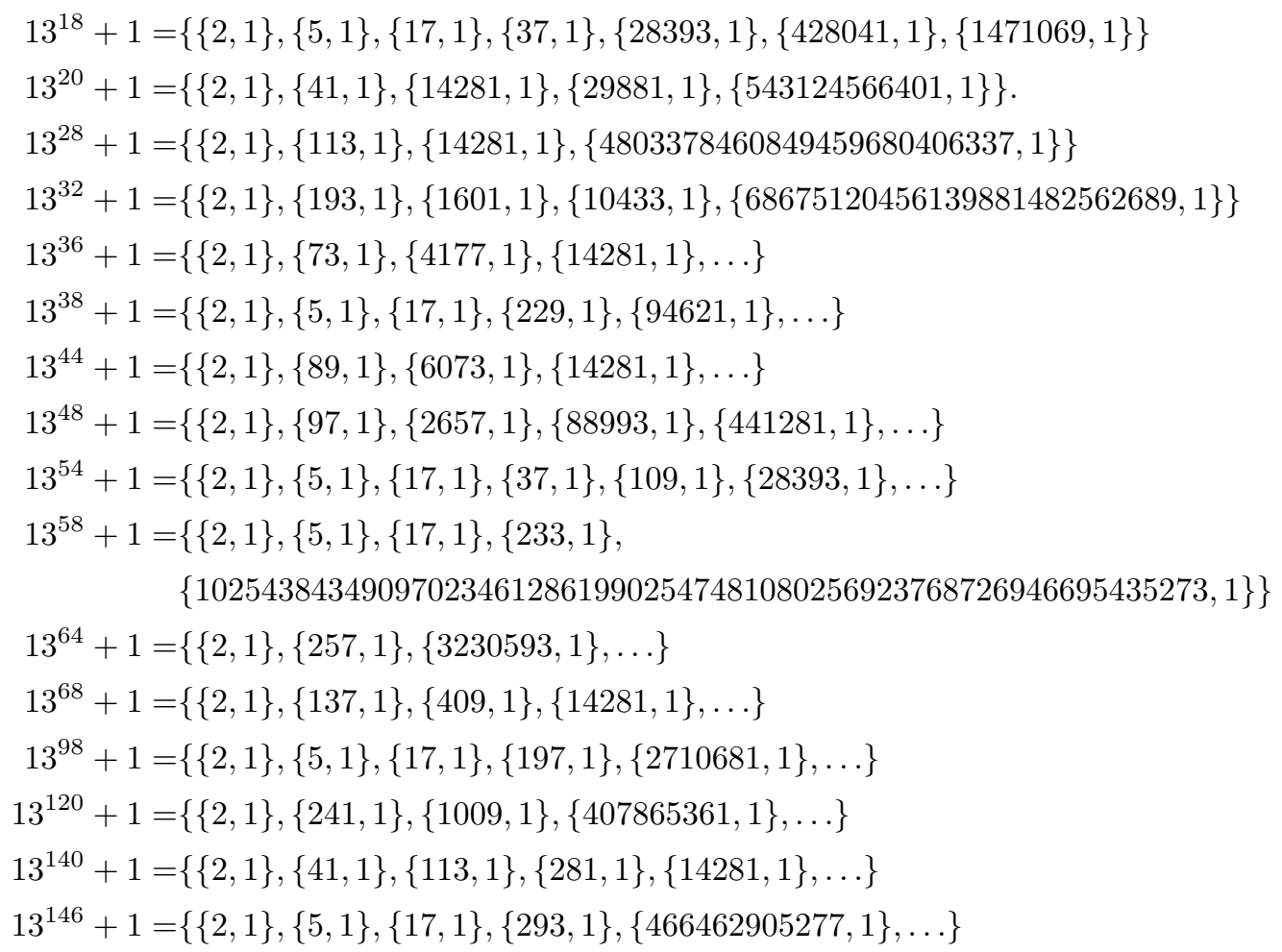




\title{
Appendix E Factors of $17^{t}-1$
}

\author{
$17^{9}-1=\{\{2,4\},\{19,1\},\{307,1\},\{1270657,1\}\}$ \\ $17^{19}-1=\{\{2,4\},\{229,1\},\{1103,1\},\{202607147,1\},\{291973723,1\}\}$ \\ $17^{21}-1=\{\{2,4\},\{43,1\},\{307,1\},\{13567,1\},\{25646167,1\},\{940143709,1\}\}$ \\ $17^{23}-1=\{\{2,4\},\{47,1\},\{26552618219228090162977481,1\}$ \\ $17^{27}-1=\{\{2,4\},\{19,1\},\{307,1\},\{433,1\},\{24733,1\},\{1270657,1\}, \ldots\}$ \\ $17^{29}-1=\{\{2,4\},\{59,1\},\{7193,1\},\{6088087,1\},\{11658852700685942029849,1\}\}$ \\ $17^{33}-1=\{\{2,4\},\{67,1\},\{307,1\},\{3697,1\},\{976669,1\}, \ldots\}$ \\ $17^{37}-1=\{\{2,4\},\{149,1\},\{223,1\},\{1016919604559540581,1\}, \ldots\}$ \\ $17^{39}-1=\{\{2,4\},\{157,1\},\{307,1\},\{212057,1\},\{2919196853,1\}, \ldots\}$ \\ $17^{41}-1=\{\{2,4\},\{83,1\},\{892079,1\},\{13365673,1\}, \ldots\}$ \\ $17^{49}-1=\{\{2,4\},\{491,1\},\{883,1\},\{25646167,1\},\{474969439337,1\}, \ldots\}$ \\ $17^{51}-1=\{\{2,4\},\{103,1\},\{307,1\},\{409,1\},\{10949,1\},\{1749233,1\}, \ldots\}$ \\ $17^{63}-1=\{\{2,4\},\{19,1\},\{43,1\},\{127,1\},\{307,1\},\{13567,1\}, \ldots\}$ \\ $17^{73}-1=\{\{2,4\},\{293,1\},\{1621745371,1\},\{3038535503,1\},\{319344640907,1\}, \ldots\}$ \\ $17^{75}-1=\{\{2,4\},\{151,1\},\{307,1\},\{2551,1\},\{5101,1\},\{5351,1\}, \ldots\}$ \\ $17^{89}-1=\{\{2,4\},\{179,1\},\{7121,1\},\{10859,1\}, \ldots\}$ \\ $17^{95}-1=\{\{2,4\},\{191,1\},\{229,1\},\{1103,1\},\{88741,1\},\{202607147,1\}, \ldots\}$ \\ $17^{97}-1=\{\{2,4\},\{389,1\},\{90976939813,1\},\{65888627940954399173,1\}, \ldots\}$ \\ $17^{119}-1=\{\{2,4\},\{239,1\},\{2381,1\},\{3571,1\},\{10949,1\},\{16661,1\}, \ldots\}$ \\ $17^{127}-1=\{\{2,4\},\{509,1\},\{2287,1\},\{19813,1\},\{9085073,1\}, \ldots\}$ \\ $17^{131}-1=\{\{2,4\},\{263,1\},\{367056542472353396414551932367550703732602240$ \\ 626266437580589512042557939674013046425712329694554361136410 \\ $49586841689181084276511163513402458984276636720387829,1\}\}$ \\ $17^{135}-1=\{\{2,4\},\{19,1\},\{271,1\},\{307,1\},\{433,1\},\{3691,1\},\{24733,1\}, \ldots\}$ \\ $17^{165}-1=\{\{2,4\},\{67,1\},\{307,1\},\{331,1\},\{3697,1\},\{46861,1\},\{88741,1\}, \ldots\}$ \\ $17^{179}-1=\{\{2,4\},\{359,1\},\{18617,1\},\{121721,1\},\{1108776121,1\}, \ldots\}$ \\ $17^{191}-1=\{\{2,4\},\{383,1\},\{3738211891,1\}, \ldots\}$ \\ $17^{221}-1=\{\{2,4\},\{443,1\},\{10949,1\},\{151607,1\},\{212057,1\},\{1749233,1\}, \ldots\}$ \\ $17^{233}-1=\{\{2,4\},\{467,1\}$, \\ $\{662463291227225180212676697073783578164677575256855318635602076$ \\ 6775492769444675372228864152379392277413358880893612987903057911321843 \\ 4295120243879391825646598579562732265304498078847938955250726380620973 \\ 1783877309393965931437452025559757828597962954773024783566035419681902 \\ $8096943763,1\}\}$
}




\section{Appendix F Factors of $17^{t}+1$}

$$
\begin{aligned}
17^{12}+1 & =\{\{2,1\},\{73,1\},\{1321,1\},\{41761,1\},\{72337,1\}\} \\
17^{16}+1 & =\{\{2,1\},\{257,1\},\{1801601,1\},\{52548582913,1\}\} \\
17^{18}+1 & =\{\{2,1\},\{5,1\},\{29,1\},\{37,1\},\{109,1\},\{181,1\},\{2089,1\},\{83233,1\}, \ldots\} \\
17^{20}+1 & =\{\{2,1\},\{41,1\},\{41761,1\},\{1186844128302568601,1\}\} \\
17^{22}+1 & =\{\{2,1\},\{5,1\},\{29,1\},\{89,1\},\{25741,1\}, \ldots\} \\
17^{30}+1 & =\{\{2,1\},\{5,2\},\{29,1\},\{61,1\},\{541,1\},\{21881,1\}, \ldots\} \\
17^{34}+1 & =\{\{2,1\},\{5,1\},\{29,1\},\{137,1\},\{1361,1\},\{2698649,1\}, \ldots\} \\
17^{40}+1 & =\{\{2,1\},\{241,1\},\{18913,1\},\{184417,1\}, \ldots\} \\
17^{44}+1 & =\{\{2,1\},\{353,1\},\{41761,1\},\{4578289,1\}, \ldots\} \\
17^{48}+1 & =\{\{2,1\},\{97,1\},\{257,1\},\{1120513,1\},\{1801601,1\},\{53160769,1\}, \ldots\} \\
17^{56}+1 & =\{\{2,1\},\{113,1\},\{337,1\},\{18913,1\},\{184417,1\}, \ldots\} \\
17^{66}+1 & =\{\{2,1\},\{5,1\},\{29,1\},\{89,1\},\{397,1\},\{19801,1\}, \ldots\} \\
17^{70}+1 & =\{\{2,1\},\{5,2\},\{29,1\},\{281,1\},\{21881,1\},\{63541,1\}, \ldots\} \\
17^{86}+1 & =\{\{2,1\},\{5,1\},\{29,1\},\{173,1\},\{2237,1\},\{26673589,1\}, \ldots\} \\
17^{96}+1 & =\{\{2,1\},\{193,1\},\{1409,1\},\{165569,1\},\{2533128442908097,1\}, \ldots\} \\
17^{98}+1 & =\{\{2,1\},\{5,1\},\{29,1\},\{197,1\},\{578789,1\},\{5766433,1\}, \ldots\} \\
17^{116}+1 & =\{\{2,1\},\{233,1\},\{41761,1\},\{244297,1\}, \ldots\} \\
17^{134}+1 & =\{\{2,1\},\{5,1\},\{29,1\},\{269,1\},\{522580700249,1\}, \ldots\} \\
17^{138}+1 & =\{\{2,1\},\{5,1\},\{29,1\},\{277,1\},\{83233,1\},\{102121,1\}, \ldots\} \\
17^{156}+1 & =\{\{2,1\},\{73,1\},\{313,1\},\{1321,1\},\{41761,1\},\{72337,1\}, \ldots\} \\
17^{158}+1 & =\{\{2,1\},\{5,1\},\{29,1\},\{317,1\},\{6637,1\},\{155473,1\}, \ldots\} \\
17^{200}+1 & =\{\{2,1\},\{241,1\},\{401,1\},\{18913,1\},\{184417,1\},\{3583912721,1\}, \ldots\} \\
17^{224}+1 & =\{\{2,1\},\{449,1\},\{1409,1\},\{165569,1\}, \ldots\}
\end{aligned}
$$

